\title{
L'activité rémunérée des étudiants et ses liens avec la réussite des études
}

Les enseignements des enquêtes Emploi 2013-2015

Student Employment and Its Links to Academic Achievement. Lessons from the

French Labour Force Surveys 2013-2015

\section{Sandra Zilloniz}

\section{OpenEdition}

\section{Journals}

Édition électronique

URL : http://journals.openedition.org/travailemploi/7776

DOI : 10.4000/travailemploi.7776

ISSN : $1775-416 \mathrm{X}$

\section{Éditeur}

DARES - Ministère du Travail

\section{Édition imprimée}

Date de publication : 1 octobre 2017

Pagination : 89-117

ISSN : 0224-4365

\section{Référence électronique}

Sandra Zilloniz, «L'activité rémunérée des étudiants et ses liens avec la réussite des études », Travail et Emploi [En ligne], 152 | octobre-décembre 2017, mis en ligne le 11 juillet 2019, consulté le 10 décembre 2020. URL : http://journals.openedition.org/travailemploi/7776 ; DOI : https://doi.org/ 10.4000/travailemploi.7776 


\title{
L'activité rémunérée des étudiants et ses liens avec la réussite des études
}

\section{Les enseignements des enquêtes Emploi 2013-2015}

\author{
Sandra Zilloniz
}

Les emplois étudiants revêtent de nombreuses formes. L'un des critères centraux pour les différencier est leur lien ou non avec les études suivies. Parmi les étudiants qui travaillent, plus de la moitié exerce une activité prévue par leurs études (stage, apprentissage, internat de médecine, etc.), d'après les enquêtes Emploi 2013-2015 de l'Insee. Les autres exercent une activité sans lien avec leurs études, occasionnellement ou régulièrement tout au long de l'année. Celle-ci correspond à des emplois moins qualifiés et plus souvent à temps partiel que lorsque le lien avec les études est avéré, ce qui n'exclut pas qu'elle puisse représenter une charge horaire lourde et contraignante vis-à-vis de l'emploi du temps studieux et freiner la réussite des études. Les enfants d'ouvriers, ayant plus souvent que les autres un emploi étudiant non lié aux études et dont la réussite dans l'enseignement supérieur est souvent présentée comme fragile, seraient ainsi particulièrement pénalisés.

\begin{abstract}
A ujourd'hui très nombreux, les étudiants représentaient jusqu'au milieu du $\mathrm{Xx}^{\mathrm{e}}$ siècle une faible part de la jeunesse et étaient majoritairement des garçons issus des classes supérieures (REY, 2009). La multiplication par cinq des effectifs étudiants entre 1960 et aujourd'hui a transformé le paysage de l'enseignement supérieur, puisqu'elle s'est accompagnée d'une diversification des profils et des conditions de vie des étudiants (DuBET, 1994 ; GALland, 1995 ; ERLICH, 1998 ; LAHIRE, 1997 ; GIRET et al., 2016) ainsi que d'une évolution du poids relatif des filières et des disciplines au sein des établissements. L'augmentation des effectifs s'est en effet accompagnée d'une croissance des orientations des étudiants vers les formations non universitaires et les
\end{abstract}

\footnotetext{
* Ministère du Travail, Direction de l'animation de la recherche, des études et des statistiques (Dares) (au moment de la rédaction de l'article); sandra.zilloniz@finances.gouv.fr.
} 
cursus à vocation professionnalisante ${ }^{1}$, destinés à faire face aux mutations du marché du travail et aux attentes d'une population étudiante de plus en plus diversifiée (DuRUBELLAT, VERLEY, 2009). L'allongement des scolarités a, par ailleurs, incontestablement bouleversé les trajectoires des jeunes vers l'âge adulte, prolongeant la jeunesse : les âges au premier emploi, à la décohabitation familiale, au mariage ou au premier enfant sont plus tardifs aujourd'hui que dans les années 1960. La démocratisation de l'accès à l'enseignement, concomitante à la massification, signifie aussi que la question du financement des études - éventuellement assuré par l'emploi étudiant - s'est posée pour davantage d'étudiants (REY, 2009). Les situations de cumul emploi-études chez les jeunes, en particulier lorsque ces emplois ne sont pas liés aux études, sont favorisées par un marché du travail où les contrats proposés aux jeunes sont souvent de courte durée - contrats d'intérim, contrats à temps partiel et emplois peu qualifiés (GALTIER, MinNI, 2015). Les étudiants constituent de ce point de vue une main-d'œuvre peu coûteuse, relativement flexible et rapidement opérationnelle, prête à occuper des emplois peu qualifiés (PINTO, 2014).

Le nombre d'étudiants en emploi est cependant difficile à mesurer. D'une part, la situation des jeunes sur le marché du travail varie fortement selon l'âge (GALTIER, MinNI, 2015). D'autre part, le taux d'emploi des étudiants et les formes qu'il prend varient au cours de l'année en fonction du calendrier des études : plus faible en début d'année scolaire, il est plus intense au printemps, en raison des stages, et pendant les vacances d'été du fait des emplois occasionnels (COUDIN, TAVAN, 2008).

L'emploi étudiant recouvre ainsi une diversité de situations. Dans la littérature, cette diversité conduit les auteurs à proposer des typologies d'emploi étudiant, qui ont en commun de distinguer les activités professionnelles selon qu'elles sont intégrées ou non dans un cursus de formation (Thiphaine, 2002 ; Gruel, Thiphaine, 2004 ; Belghith, 2015 ; Giret, 2011 ; Coudin, Tavan, 2008 ; Béduwé, Giret, 2004). Les raisons avancées par les étudiants pour exercer une activité sont également très variées. Si la principale motivation est financière (Ove, 2016), l'emploi se justifie aussi par la recherche d'indépendance vis-à-vis des parents (ECKERT, 2009) ou encore, parfois, par le souhait d'acquérir une expérience professionnelle.

En France, les formations sont construites pour des étudiants à temps plein et ceux qui exercent une activité rémunérée parallèle à leurs études ont généralement droit à peu d'aménagement. Compte tenu de la contrainte de temps qu'elles peuvent imposer et de la variété des formes qu'elles peuvent prendre, les activités exercées ont des influences diverses sur les parcours d'études. Les activités intégrées aux études (apprentissage, internat de médecine, stage, etc.) ne sont, en général, pas considérées comme pénalisantes pour la réussite de celles-ci, alors que les activités qui entrent en

1. Le développement des formations universitaires professionnalisantes prend son essor dans les années 1970 et se poursuit dans les années 1980, avec notamment la création à l'université de ses propres écoles d'ingénieurs et de filières d'excellence pouvant rivaliser avec les grandes écoles. En outre, le passage au LMD (Licence Master Doctorat) au début des années 2000 entraîne une reconfiguration de l'offre de formation à l'université dans le sens d'une professionnalisation des parcours (licence et master professionnels). 
concurrence avec les études le sont davantage (Gruel, Thiphaine, 2004 ; BELGHITH, 2015). $18 \%$ des étudiants qui exercent une activité non intégrée estiment ainsi qu'elle a un impact négatif sur leurs résultats d'études (Ove, 2016). Une activité professionnelle non liée à la formation peut en effet réduire de manière non négligeable le temps consacré aux études (cours, révisions), avoir un impact négatif sur les performances scolaires des étudiants et donc augmenter le risque d'échec et de décrochage. Dans la littérature française et nord-américaine, il existe un consensus sur le fait que le temps de travail de l'emploi est déterminant pour la réussite des études mais il n'y a pas d'accord sur la détermination du seuil horaire à partir duquel le travail aurait un impact négatif sur les études : 20 heures par semaine selon Christopher J. RuHM (1997), 15 heures pour Marcel Dagenais et ses co-auteurs (2000), Claude MonTMARQueTte et ses coauteurs (2007) ou encore Catherine BÉDUwÉ et Jean-François GIRET (2004), 16 heures pour Magali BefFy et ses co-auteurs (2009) et 10 heures d'après Daniel PARENT (2006). L'un des enjeux du travail présenté ici est de questionner, à partir des données issues des enquêtes Emploi de 2013 à 2015, les liens entre les caractéristiques de l'emploi étudiant et la réussite des études. On s'interrogera également sur le lien entre travail étudiant et origine sociale des étudiants. Les étudiants d'origine populaire, plus nombreux qu'hier, exercent en effet plus souvent une activité rémunérée non liée à leurs études (BELGHith, 2015). Or, leurs parcours d'études et leur réussite dans l'enseignement supérieur paraissent relativement contrastés et largement dépendants de leurs acquis dans l'enseignement secondaire et primaire (Poullaouec, Hugrée, 2011).

La première partie de l'article présente les données. La deuxième partie introduit une typologie d'emplois étudiants : sur le modèle des travaux existants sur le sujet, nous distinguons notamment les emplois selon qu'ils sont ou non intégrés aux études, y compris lorsqu'il s'agit d'activités exercées uniquement l'été. Quelle est l'organisation du travail selon les types d'activités définis et quels étudiants sont concernés ? Nous nous appuyons sur ces analyses pour, dans une troisième partie, explorer la relation existante entre emploi étudiant et réussite universitaire. Quels sont les liens entre les caractéristiques de l'emploi étudiant - son intensité, son articulation éventuelle avec les études - et la réussite aux examens ?

\section{Les données : atouts et limites de l'enquête Emploi pour l'analyse de l'emploi étudiant}

Dans cette étude, nous mobilisons les données 2013, 2014 et 2015 de l'enquête Emploi de l'Insee (encadré 1). L'enquête Emploi n'est spécialisée ni sur les jeunes ni sur les étudiants, mais elle présente, par rapport à d' autres sources, plusieurs avantages pour la mesure de l'activité étudiante. Réalisée en continu, elle fournit une information sur l'emploi à un instant donné, recense tous les types d'emplois (réguliers ou occasionnels), prend en compte la temporalité et donc la saisonnalité de l'emploi au cours de l'année, particulièrement importante pour comprendre l'emploi étudiant et, enfin, 


\section{ENCADRÉ 1}

\section{L'enquête Emploi de l'Insee}

Depuis 2003, l'enquête Emploi est réalisée en continu tout au long de l'année en France métropolitaine. Cette enquête est réalisée par l'Insee auprès des ménages vivant dans des logements ordinaires. Ainsi, les communautés (foyers, cités universitaires, hôpitaux, maisons de retraite, prisons) ne sont pas enquêtées. Échappant à cette règle, les internats sont intégrés au champ de l'enquête. Les élèves ou étudiants habitant dans un logement indépendant (hors cité universitaire ou foyer d'étudiants) sont rattachés à leur logement s'ils le considèrent comme leur logement principal, ou au logement de leurs parents s'ils y retournent fréquemment, notamment pendant les vacances scolaires. De même, une personne ayant un logement occasionnel mais retournant le week-end dans son foyer principal est rattachée à ce dernier. Cela a un impact sur le mode de logement renseigné dans l'enquête. Ainsi, la part d'étudiants qui vivent dans un logement indépendant de leurs parents est plus faible que celle pouvant être observée dans l'enquête Conditions de vie des étudiants de l'Observatoire national de la vie étudiante (OVE) qui renseigne, quant à elle, sur le logement occupé pendant une semaine normale de cours, avec $39 \%$ d'étudiants en logement indépendant en moyenne de 2013 à 2015 dans l'enquête Emploi contre $68 \%$ dans l'enquête Conditions de vie (OvE, 2016).

elle offre la possibilité de suivre un échantillon d'étudiants sur 5 trimestres et donc d'étudier leur parcours pendant les études et leur réussite aux examens.

\section{Une enquête qui permet une mesure du cumul emploi-études}

À la question du nombre d'étudiants en France qui travaillent pendant leurs études, la littérature sur le sujet répond par quasiment autant de chiffres différents que de sources permettant sa mesure (tableau A en annexe). Cela révèle la complexité de la mesure du travail étudiant, qui varie selon que l'on intègre toutes les situations d'emploi (apprentissage, stages, jobs d'été) ou que l'on considère le travail effectué au moins une fois dans l'année ou à une date donnée.

Les enquêtes Génération du Centre d'études et de recherches sur l'emploi et les qualifications (Céreq) interrogent de façon rétrospective les jeunes sortants de formation initiale sur leurs emplois éventuellement exercés pendant leurs études, et sur les formes prises par ces expériences professionnelles : emplois réguliers (activités salariées d'au moins huit heures par semaine), apprentissage, emplois occasionnels (petits boulots) ou stages. Ces enquêtes ont l'avantage de capter l'ensemble des emplois des étudiants au cours de leurs études - ce que ne fait pas l'enquête Emploi - mais elles ont plusieurs inconvénients. Tout d'abord, elles font appel à la mémoire des individus, qui peut être plus ou moins fiable. En outre, les caractéristiques des emplois occupés pendant les études ne sont pas décrites - à l'exception des emplois réguliers - et le moment où ils se sont déroulés n'est pas renseigné, sauf lorsqu'il 
s'agit d'emplois réguliers conservés après l'arrêt des études. Les enquêtes Génération permettent surtout d'analyser le lien entre l'emploi pendant les études et l'insertion professionnelle, moins celui pouvant exister avec la réussite des études (voir l'analyse de Catherine Beduwé et Jean-François GiRet [2004] et celle de Sabina Issennane [2011] centrée sur l'apprentissage). Enfin, ces enquêtes effectuent une mesure très particulière de l'activité, qui prend en compte les emplois occasionnels au même titre que les emplois réguliers et ne permet pas de calculer des taux d'emploi des étudiants pouvant être comparés à ceux d'autres populations.

La façon dont les enquêtes sont construites et les questions posées influencent également les réponses. L'enquête Emploi en continu (depuis 2003) retient à ce titre la définition de l'activité du Bureau international du travail (BIT) ${ }^{2}$ et classe les individus en emploi dès l'instant qu'ils ont travaillé au moins une heure pendant la semaine de référence. Or, avant la refonte de l'enquête Emploi (effective après 2002), on pouvait

\section{ENCADRÉ 2}

\section{La construction des échantillons}

Pour la description des activités exercées en cours d'études, les données des enquêtes Emploi sont empilées sur tous les trimestres des années 2013, 2014 et 2015. À partir de ces données, différentes populations sont sélectionnées :

- les élèves ou étudiants de 18 à 29 ans qui suivent une formation dans le supérieur : 46125 étudiants (graphiques 1 et 2);

- les élèves ou étudiants de 18 à 29 ans qui suivent une formation dans le supérieur et occupent un emploi au moment de l'enquête (au cours d'une semaine de référence) : 10155 étudiants (tableau 1, tableau 2, tableau 3).

Pour l'analyse de la probabilité d'exercer une activité rémunérée (tableau B, en annexe), les modèles ne portent que sur les étudiants interrogés pour la première fois dans l'enquête, afin d'éviter le biais lié à l'interrogation répétée des individus dans l'enquête. Dans un souci de cohérence avec l'étude des parcours étudiants, les élèves de CPGE et les étudiants en doctorat sont exclus de ces analyses. L'échantillon utilisé est composé de 10290 étudiants (modèle 1) et de 2277 étudiants en emploi (modèle 2).

Pour l'étude des parcours étudiants, on construit un panel d'étudiants. Pour cela, on utilise les données portant sur les jeunes de 18 à 29 ans issues des bases trimestrielles des enquêtes Emploi. On sélectionne les jeunes de 18 à 29 ans interrogés au deuxième trimestre de l'année et réinterrogés au troisième et quatrième trimestre de la même année (pour 2013, 2014 et 2015). Les bases sont appariées deux à deux (T2-T3/T3-T4 pour chaque année), puis sont de nouveau appariées pour l'année, de sorte à retrouver les informations

2. Les actifs occupés regroupent toutes les personnes ayant exercé une activité rémunérée au moins une heure au cours de la semaine précédant l'interrogation du ménage (ou qui travaillent habituellement mais sont en congé, en formation, en arrêt maladie ou maternité). Parmi les personnes qui ne sont pas comptabilisées comme actifs occupés, celles qui recherchent activement un emploi et qui sont disponibles pour travailler sont comptabilisées comme chômeurs. Enfin, les personnes qui ne sont ni actives occupées ni chômeuses sont classées en tant qu'inactives. 
sur le parcours des étudiants entre le deuxième trimestre de l'année et les deux trimestres qui suivent (la validation de l'année étant généralement connue entre le troisième et le quatrième trimestre de l'année). Les bases sont par la suite empilées pour les trois années et les individus suivant une formation dans l'enseignement supérieur (hors doctorat et élèves de CPGE, cas particuliers quant au rapport aux études et trop peu nombreux pour être isolés) âgés de 18 à 29 ans au deuxième trimestre de chaque année sont sélectionnés. Le panel d'étudiants ainsi constitué comprend 4369 individus (tableau 4, modèle 1). À partir de ce panel, on sélectionne pour des analyses les seuls étudiants en emploi qui forment une population de 907 étudiants (tableau 4, modèles 2 et 3).

\section{TABLEAU - Construction du panel d'étudiants interrogés au deuxième trimestre de l'année n et réinterrogés aux troisième et quatrième trimestres de l'année n}

\begin{tabular}{|c|c|c|c|c|c|c|c|c|}
\hline & 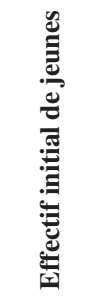 & 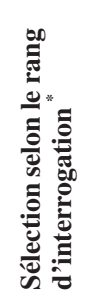 & 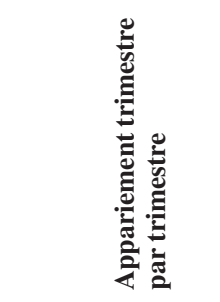 & & 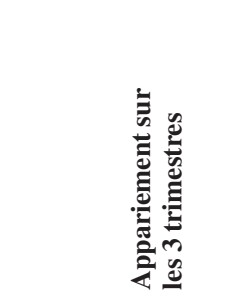 & & 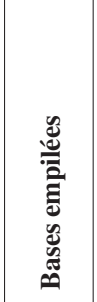 & 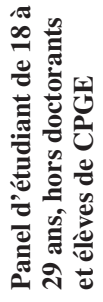 \\
\hline $2^{\mathrm{e}}$ trimestre 2013 & 25960 & 22986 & \multirow{2}{*}{$\begin{array}{l}2^{\mathrm{e}} \text { trimestre } 2013- \\
3^{\mathrm{e}} \text { trimestre } 2013\end{array}$} & \multirow{2}{*}{17256} & \multirow{4}{*}{$\begin{array}{c}2^{\mathrm{e}} \text { trimestre } 2013- \\
4^{\mathrm{e}} \text { trimestre } 2013\end{array}$} & \multirow{4}{*}{11968} & \multirow{12}{*}{35345} & \multirow{12}{*}{4369} \\
\hline $3^{\mathrm{e}}$ trimestre 2013 & 24100 & 18070 & & & & & & \\
\hline $3^{\mathrm{e}}$ trimestre 2013 & 24100 & 21489 & $3^{\mathrm{e}}$ trimestre 2013 - & 16782 & & & & \\
\hline $4^{\mathrm{e}}$ trimestre 2013 & 24728 & 18155 & HIIIESTIE 2015 & & & & & \\
\hline $2^{\mathrm{e}}$ trimestre 2014 & 24898 & 22646 & \multirow{2}{*}{$\begin{array}{c}2^{\mathrm{e}} \text { trimestre } 2014- \\
3^{\mathrm{e}} \text { trimestre } 2014\end{array}$} & \multirow{2}{*}{16801} & \multirow{4}{*}{$\begin{array}{l}2^{\mathrm{e}} \text { trimestre } 2014- \\
4^{\mathrm{e}} \text { trimestre } 2014\end{array}$} & \multirow{4}{*}{11784} & & \\
\hline $3^{\mathrm{e}}$ trimestre 2014 & 23896 & 17614 & & & & & & \\
\hline $3^{\mathrm{e}}$ trimestre 2014 & 23896 & 21703 & \multirow{2}{*}{$\begin{array}{l}3^{\mathrm{e}} \text { trimestre } 2014- \\
4^{\mathrm{e}} \text { trimestre } 2014\end{array}$} & \multirow{2}{*}{16785} & & & & \\
\hline $4^{\mathrm{e}}$ trimestre 2014 & 24565 & 18041 & & & & & & \\
\hline $2^{\mathrm{e}}$ trimestre 2015 & 24307 & 21948 & $2^{\mathrm{e}}$ trimestre $2015-$ & & \multirow{4}{*}{$\begin{array}{l}2^{\mathrm{e}} \text { trimestre } 2015- \\
4^{\mathrm{e}} \text { trimestre } 2015\end{array}$} & \multirow{4}{*}{11593} & & \\
\hline $3^{\mathrm{e}}$ trimestre 2015 & 23342 & 17249 & 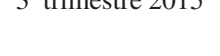 & & & & & \\
\hline $3^{\mathrm{e}}$ trimestre 2015 & 23342 & 21161 & \multirow{2}{*}{$\begin{array}{l}3^{\mathrm{e}} \text { trimestre } 2015- \\
4^{\mathrm{e}} \text { trimestre } 2015\end{array}$} & \multirow{2}{*}{16485} & & & & \\
\hline $4^{\mathrm{e}}$ trimestre 2015 & 24132 & 17704 & & & & & & \\
\hline
\end{tabular}

* Rang d'interrogation de 1 à 5 pour le premier trimestre et de 2 à 6 pour le dernier trimestre pris en compte dans l'appariement. Champ : jeunes de 18 à 29 ans.

Source: Insee, enquêtes Emploi de 2013 à 2015. 
comparer le niveau d'emploi selon cette définition relativement extensive du BIT, avec celui issu de l'auto-classement des personnes. On dénombrait ainsi dans l'enquête Emploi de $2001^{3}$ davantage d'étudiants en emploi au sens du BIT que d'après l'autoclassement des individus (GonZalez-Demichel, NauZe-Fichet, 2003). Certains étudiants classés actifs occupés au sens du BIT se déclaraient en effet inactifs, alors qu'ils avaient travaillé au moins une heure la semaine de référence.

L'étude exploite les enquêtes Emploi de 2013, 2014 et 2015, empilées afin d'obtenir des résultats plus robustes (encadré 2). L'activité est définie au sens du BIT. Les élèves et étudiants actifs occupés sont ici les jeunes de 18 à 29 ans qui suivent une formation conduisant à un diplôme ou à un titre reconnus de l'enseignement supérieur (y compris en apprentissage) et qui occupent un emploi (y compris un stage) au cours d'une semaine dite de référence ${ }^{4}$. Selon ces concepts, en moyenne, de 2013 à 2015 , parmi les 2,3 millions d'étudiants du supérieur, $23 \%$ sont actifs occupés. L'exercice d'une activité en cours d'études est plus fréquent qu'il y a dix ans, principalement en raison du développement de l'apprentissage. En moyenne sur 2004-2006, parmi les 2,1 millions d'étudiants du supérieur, $19 \%$ avaient un emploi (Coudin, TAVAN, 2008).

Notre typologie des emplois étudiants distingue les activités intégrées (ou liées) aux études des autres : les activités intégrées aux études sont incluses dans la formation et nécessaires à l'obtention du diplôme, et donc en lien direct avec les études. Sur le modèle de ce qu' ont fait Élise Coudin et Chloé TAVAN (2008) sur les enquêtes Emploi de 2004 à 2006, nous considérons que cela concerne l'ensemble des étudiants en stage ou en apprentissage. À ces cas de figure, nous ajoutons les Attachés temporaires d'enseignement et de recherche (Ater), ainsi que les internes et les collaborateurs de recherche, ces trois catégories étant appelées dans la suite de l'étude «salariés étudiants ». Ce classement est rendu possible par le rapprochement de la profession exercée avec les études suivies (tableau 1). L'activité non liée aux études est définie, par opposition, comme toute activité rémunérée qui ne fait pas partie de la formation ou qui en est éloignée de par son contenu. Nous distinguons pour les activités intégrées aux études comme pour celles qui ne le sont pas, celles qui sont exercées occasionnellement et celles qui sont exercées régulièrement ${ }^{5}$.

\section{Une enquête qui donne une mesure de la réussite dans le supérieur}

On peut généralement mesurer la réussite des études par l'obtention d'un diplôme ou la validation totale ou partielle d'une année d'études. Les données de l'enquête Emploi, où les individus sont, sauf attrition, interrogés six trimestres consécutifs,

\footnotetext{
3. L'enquête Emploi en continu ne permet plus de faire cette comparaison, la mesure spontanée de l'emploi à partir des déclarations des enquêtés n'y figurant plus.

4. Les données recueillies sur l'ensemble des semaines sont ainsi représentatives de l'année.

5. Pour ceux qui ont une seule activité professionnelle (96\%), la question dans l'enquête Emploi est : « S'agit-il d'une activité régulière, ou bien plutôt d'une activité temporaire ou d'appoint (ex : job d'été, travail purement occasionnel ou peu fréquent - fréquence moins qu'hebdomadaire) ? »
} 
permettent d'étudier le passage au niveau d'études supérieur d'une année universitaire à l'autre. La simultanéité des événements et les interrogations successives permettent par ailleurs d'intégrer l'ensemble des étudiants, de voir s'ils ont travaillé pendant leurs études et s'ils poursuivent ou non celles-ci, ce que ne font pas les enquêtes Conditions de vie de l'OVE ou Génération du Céreq. L'OVE observe la réussite pour l'année universitaire qui précède l'enquête (mise en parallèle avec des informations sur l'activité rémunérée exercée l'année précédente) pour les étudiants inscrits l'année universitaire qui suit à l'université (ou dans un Institut universitaire technologique [IUT]), dans une Classe préparatoire aux grandes écoles (CPGE) ou une Section de technicien supérieur (STS) publiques. Les résultats ne concernent donc pas les étudiants inscrits dans d'autres institutions, mais surtout n'intègrent pas les étudiants qui se réorientent dans un établissement non couvert par l'enquête ou ceux qui ont abandonné leurs études, ce qui rend délicat l'étude de la réussite scolaire. L'enquête Génération, quant à elle, ne permet pas d'expliquer, ni de suivre chronologiquement, emploi étudiant et retard scolaire, et comme elle concerne seulement les sortants du système éducatif, elle biaise l'étude du lien entre travail pendant les études et réussite des études (BÉDUwÉ, GIRET, 2004). L'enquête Emploi permet de faire ce lien, mais présente à son tour des inconvénients. Comme elle n'est pas spécifique à la population étudiante, certaines informations manquent pour apprécier la réussite dans l'enseignement supérieur. Il en va ainsi de la réussite dans l'enseignement secondaire (type de baccalauréat, âge auquel il a été obtenu, mention), du degré de discipline scolaire et sociale (fréquentation de la bibliothèque, utilisation d'un agenda, hygiène de vie ou encore sélection des loisirs) (GRUEL, 2002), de la qualité de l'orientation (choisie ou par défaut) ou encore de l'obtention d'une bourse sur critères sociaux.

L'utilisation des enquêtes Emploi en panel permet d'observer les parcours des étudiants (voir l'encadré 2 pour la présentation des échantillons). Chaque trimestre, un sixième de la population enquêtée est renouvelé, ce qui implique que, sauf attrition, chaque individu est ensuite présent pendant 5 trimestres. Pour la mesure de la réussite, nous regardons si, entre une année universitaire et la suivante, l'étudiant est inscrit dans un niveau d'études supérieur ${ }^{6}$. Pour cela, nous avons construit un panel de jeunes enquêtés au deuxième trimestre de l'année et réinterrogés aux troisième et quatrième trimestres de la même année (pour 2013, 2014 et 2015).

Parmi ces 4369 individus, $69 \%$ passent à un niveau d'études supérieur l'année universitaire suivante (au troisième ou au quatrième trimestre), tous niveaux d'études et disciplines confondus, avec une validation totale ou partielle de l'année. Une autre question de l'enquête Emploi permet de mesurer la réussite au diplôme ( « Avez-vous obtenu un diplôme depuis la précédente enquête ?»), mais elle n'a pas été utilisée ici pour la mesure de la réussite, car si tous les étudiants ont une chance de passer un niveau d'études chaque année, tous les niveaux d'études ne sont pas diplômants - par

6. La question est la suivante : «Depuis la dernière enquête, êtes-vous toujours dans la même classe ou la même année d'études ? » Elle est posée à ceux qui suivent une formation formelle depuis la dernière enquête. 
exemple, seuls les niveaux bac +3 (licence), bac +5 (master) et bac +8 (doctorat) sont diplômants à l'université. Elle a néanmoins été mobilisée pour distinguer les abandons des fins d'études (10\% des personnes dans l'échantillon sont en fin d'études). Ainsi, la fin des études (réussite au diplôme sans réinscription) est comptabilisée comme un passage au niveau supérieur.

\section{La démarche adoptée pour les modèles}

Deux types de modèles ont été construits. Les premiers portent sur la probabilité d'exercer une activité rémunérée pour l'ensemble des étudiants âgés de 18 à 29 ans, en prenant les individus en première interrogation (voir encadré 2), et sur la probabilité d'exercer un type particulier d'activité rémunérée (intégrée aux études, occasionnelle ou régulière) pour les seuls étudiants qui sont en emploi ${ }^{7}$ (tableau $\mathrm{B}$ en annexe). Les corrélations entre les caractéristiques des étudiants et de leurs emplois sont analysées par des modèles de régression (probit). La méthode employée a l'avantage de mettre en évidence le lien entre une variable explicative et celle à expliquer, en contrôlant par d'autres variables explicatives. Le deuxième type de modèle (tableau 4) évalue les liens entre travail étudiant et réussite des études, c'est-à-dire les corrélations entre l'activité rémunérée exercée, considérée au deuxième trimestre de l'année, et le passage au niveau supérieur d'études la même année, pour l'ensemble des étudiants, puis seulement pour ceux qui sont en emploi. Ces modèles reposent ainsi sur deux hypothèses. La première est que l'emploi occupé au deuxième trimestre de l'année est représentatif des situations d'emploi pour l'année universitaire (hors été). La seconde est que la décision de poursuivre ses études s'opère majoritairement entre deux années universitaires et que la décision de travailler précède celle de poursuivre ses études. Dans les faits, sur ce point, la réalité est plus complexe. L'étudiant pourrait par exemple choisir de travailler car il ne compte pas poursuivre ses études.

Le fait de travailler pendant ses études - en particulier lorsque l'activité exercée ne présente pas de lien avec ces dernières - et le passage au niveau supérieur d'études peuvent être conjointement déterminés ou endogènes (BEFFY et al., 2009 ; BÉDUWÉ, GIRET, 2004). En d'autres termes, les étudiants qui travaillent pendant leurs études auraient des caractéristiques observables et inobservables qui pourraient tout autant influencer le passage à un niveau supérieur d'études. La réussite d'un étudiant dépend d'un ensemble d'éléments comme ses caractéristiques individuelles, la filière d'études, son parcours studieux et ses conditions de vie. D' autres facteurs, d'ordre plus subjectif, comme le goût pour les études, la motivation, le projet professionnel de l'étudiant, le souhait d'acquérir une indépendance financière vis-à-vis de ses parents, un logement, etc. peuvent également intervenir dans la décision de travailler, et aussi dans la poursuite des études (ou les performances scolaires, pour M. DAGENAIS et ses co-auteurs [2000]).

7. Les étudiants sans activité sont enlevés des estimations des modèles 2 et 3 , car nous incluons dans ces estimations des informations sur l'activité exercée (le temps consacré à l'activité, au travail le week-end, etc.) dont les modalités non concernées ou nulles seraient trop corrélées entre elles pour les étudiants qui ne travaillent pas. 
Les résultats obtenus à partir de modèles tenant compte de l'endogénéité et leur comparaison avec ceux de modèles équivalents mais sans contrôle de l'endogénéité (probit simples) présentent très peu de différences ${ }^{8}$. Nous avons choisi de présenter les modèles ne tenant pas compte de la possible endogénéité entre le passage au niveau d'études supérieur et le fait de cumuler ou non études et emploi, ce qui implique d'interpréter les résultats non pas comme des effets causaux mais comme des corrélations.

Les modélisations ont été faites sur le panel d'étudiants non pondéré de manière longitudinale (voir encadré 2). Comme l'enquête interroge des ménages au sein de logements, le panel, par nature, est composé d'individus n'ayant pas déménagé sur la période. Cette sélection peut introduire un biais sur les caractéristiques de la population étudiée, qui ne semble pas avoir un impact significatif sur notre échantillon puisque la comparaison des principales caractéristiques du panel avec celles de la population étudiante initiale ne montre pas de déformation structurelle importante. Des variables utilisées pour le calage de l'enquête Emploi (sexe, âge, région) ont été introduites dans les modèles. La taille de l'échantillon ne permet pas de décliner les analyses par niveau ou par type d'études. Afin de pallier en partie ce problème, nous avons contrôlé les modélisations réalisées sur l'ensemble des étudiants par niveau et par discipline d'études aussi finement que possible, compte tenu des données disponibles. La taille de l'échantillon interdit l'étude des parcours de réorientation des étudiants.

\section{Les activités étudiantes dans leur diversité}

Selon la définition du travail étudiant que nous avons retenue, entre 2013 et 2015, d'après l'enquête Emploi, en moyenne $23 \%^{9}$ des étudiants sont actifs occupés - $24 \%$ des étudiants et $22 \%$ des étudiantes. Pour les étudiants qui travaillent, les activités exercées prennent de multiples formes : activité exercée tout ou partie de l'année, uniquement l'été, en parallèle des études, activité au moins en partie liée aux études (stage, vacation dans un laboratoire, internat hospitalier, etc.), activité régulière ou occasionnelle. L'emploi étudiant constitue un type particulier d'emploi : par les formes qu'il prend et par sa saisonnalité calée sur le calendrier des études, il ne peut être assimilé à l'emploi d'un jeune sorti du système éducatif (ZILLONIZ, 2017).

Si certains étudiants travaillent systématiquement en parallèle et de manière intégrée à leurs études (apprentis, internes en médecine, stagiaires, etc.), d'autres

\footnotetext{
8. La comparaison des modèles univariés et bivariés a montré que la prise en compte de l'endogénéité est justifiée dans le cas de l'exercice d'une activité rémunérée non intégrée aux études, mais change peu les résultats par rapport à ceux des modèles univariés. Elle aboutit à une mesure plus élevée du coefficient lié à l'activité rémunérée. Les niveaux des estimations et les liens statistiques sont comparables pour toutes les autres variables explicatives.

9. À ce titre, il convient de mentionner que le travail en cours d'études est moins développé en France que dans les pays nord-américains (y compris pour les lycéens pour lesquels il constitue un mode d'épargne pour financer les études supérieures particulièrement coûteuses) (BoDY, 2014) et dans d'autres pays européens, en particulier l'Allemagne, les pays anglo-saxons et ceux du nord de l'Europe (PoulET-Coulibando, 2008). Sa faible fréquence en France serait notamment liée au type d'organisation des études et à une conception plus duale du travail et des études.
} 
peuvent avoir un emploi susceptible d'entrer en concurrence avec leurs études (caissiers, surveillants, manutentionnaires, etc.). Au sein des diverses situations de cumul emploi-études, les emplois, les salaires et la durée de travail diffèrent. Une autre diversité se superpose à celle des emplois : celle des études. L'enseignement supérieur est divisé en filières qui se différencient par le « recrutement » (origine scolaire, origine sociale, sexe, etc.), les savoirs à maîtriser, les manières d'étudier, mais aussi les exigences temporelles (MiLLET, 2000). Les types d'études et la nature spécifique des savoirs transmis forment des cadres d'apprentissage et de socialisation dans des contextes spécifiques : effectifs restreints, encadrement strict et apprentissage dans les formations les plus sélectives et professionnalisantes, public plus large et hétérogène bénéficiant de moins de contraintes dans les formations universitaires (hors santé).

Les types d'activités seront principalement décrits en fonction du sexe, de l'origine sociale et du type d'études suivies. Les écarts que l'on observe sur ces dimensions entre les différents types d'activités seront confortés par des régressions logistiques multinomiales qui modélisent le type d'activité exercée (tableau B en annexe).

\section{Plus de la moitié des activités exercées par les étudiants ont un lien avec les études suivies}

Pour $56 \%$ des étudiants actifs occupés, en moyenne entre 2013 et 2015, l'activité exercée a un lien avec les études : $29 \%$ sont en apprentissage, $9 \%$ en stage, $11 \%$ sont « salariés étudiants » et $6 \%$ ont une autre activité en lien avec les études (tableau 1).

TABLEAU 1 - La nature des liens entre emploi occupé par les étudiants et études suivies

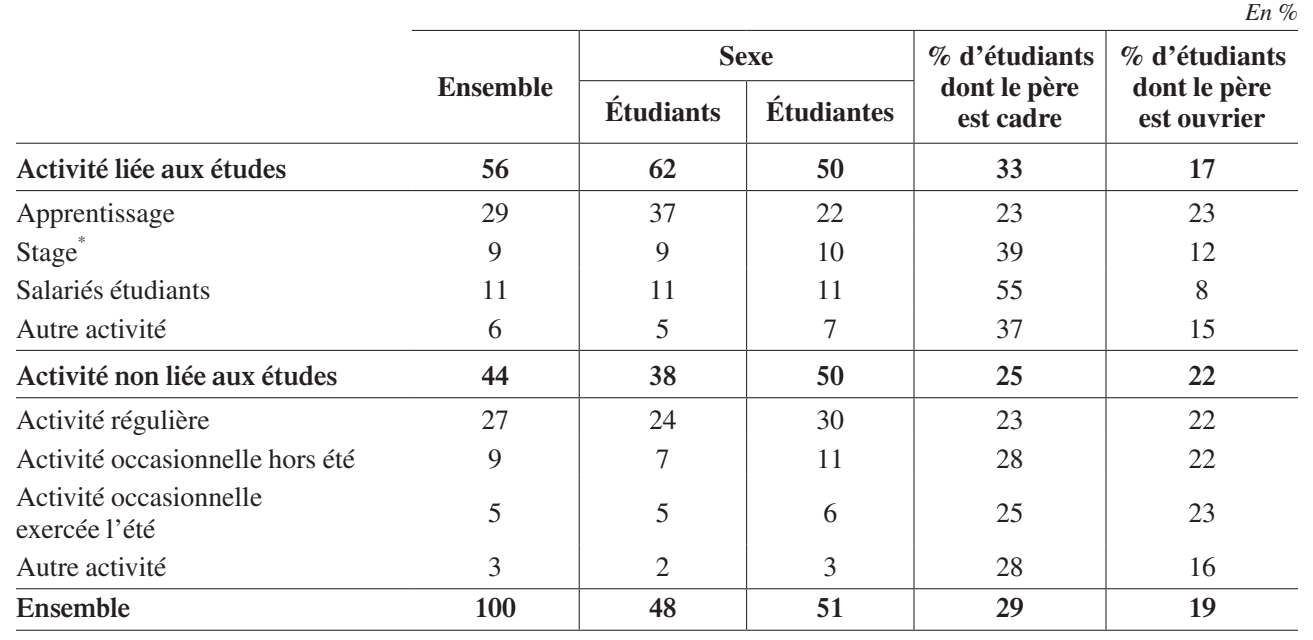

* Une faible part d'étudiants en contrat de stage a été classée ici comme salariés étudiants.

Lecture : en moyenne, de 2013 à 2015, 30 \% des étudiantes exercent une activité régulière non liée aux études.

Champ : élèves ou étudiants âgés de 18 à 29 ans qui suivent une formation formelle dans le supérieur et occupent un emploi au moment de l'enquête. Données pondérées.

Source: Insee, enquêtes Emploi de 2013 à 2015. 
GRAPHIQUE 1 - Exercice d'une activité professionnelle liée aux études selon le type d'études

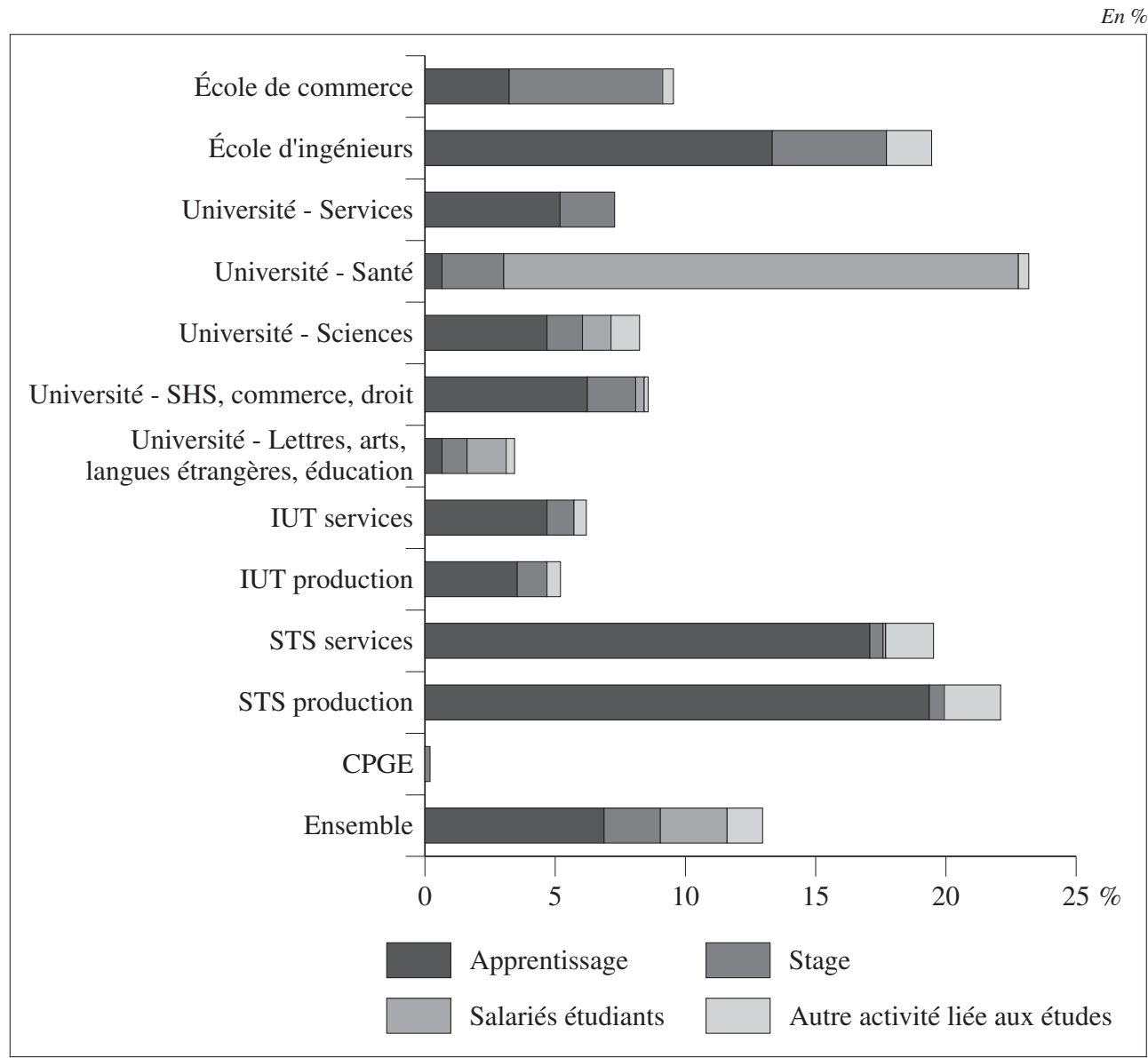

SHS : Sciences humaines et sociales ; IUT : Institut universitaire de technologie ; STS : Section de technicien supérieur ; CPGE : Classe préparatoire aux grandes écoles.

Lecture : en moyenne de 2013 à 2015, $20 \%$ des étudiants en santé à l'Université sont « salariés étudiants ».

Champ : élèves ou étudiants âgés de 18 à 29 ans qui suivent une formation formelle dans le supérieur. Données pondérées.

Source : Insee, enquêtes Emploi de 2013 à 2015.

Les activités non liées aux études (44\% de notre échantillon) peuvent être réparties dans trois groupes : les activités exercées de manière régulière $(27 \%)$, les activités occasionnelles $(14 \%)$ et les autres activités non liées aux études (3\%). Parmi les activités occasionnelles, on peut distinguer celles qui ont lieu durant l'été (au troisième trimestre de l'année).

L'apprentissage concentre $29 \%$ de l'ensemble des emplois étudiants et $53 \%$ de ceux intégrés aux études. Il est l'emploi le plus fréquent quel que soit le trimestre. Depuis la loi Séguin du 23 juillet 1987, l'apprentissage s'est progressivement ouvert à tous les niveaux de formation après avoir été longtemps cantonné à la préparation au 
GRAPHIQUE 2 - Exercice d'une activité professionnelle liée aux études selon le niveau d'études

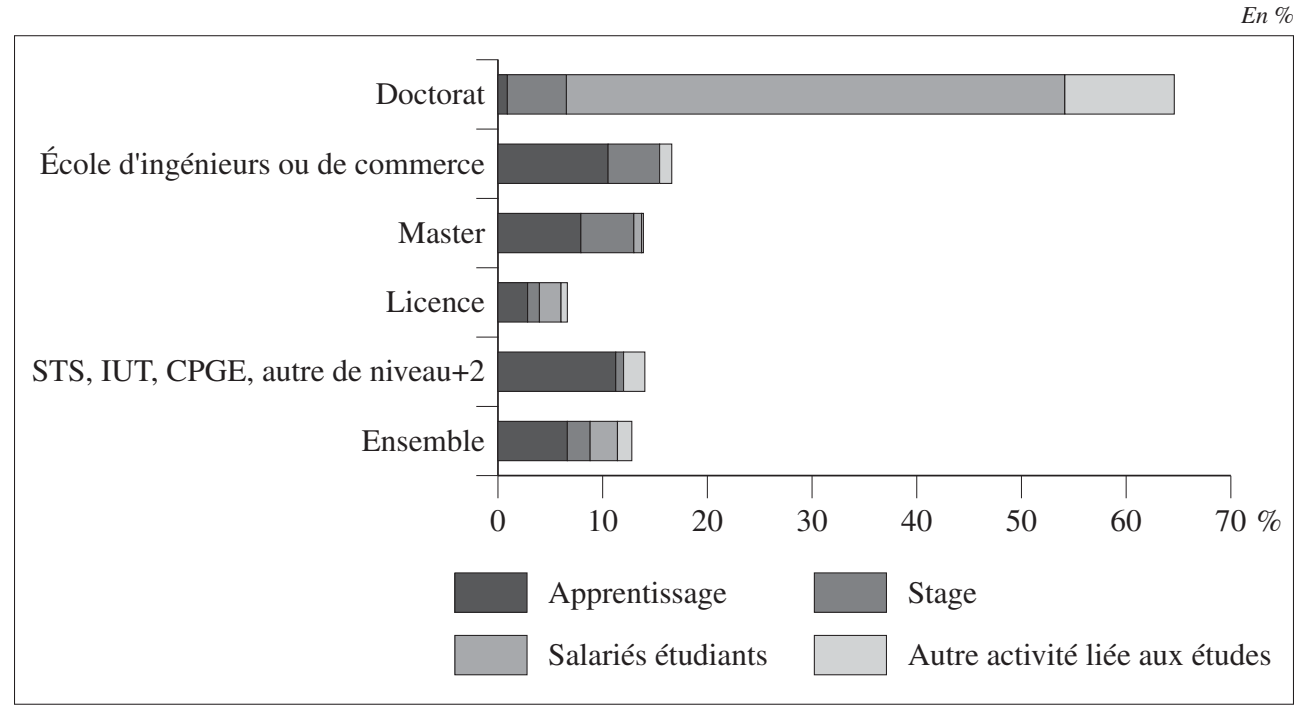

STS : Section de technicien supérieur ; IUT : Institut universitaire de technologie ; CPGE : Classe préparatoire aux grandes écoles. Pour les écoles d'ingénieurs et de commerce, le niveau d'études n'est pas connu.

Lecture : en moyenne de 2013 à 2015, 47 \% des étudiants en doctorat sont « salariés étudiants ».

Champ : élèves ou étudiants âgés de 18 à 29 ans qui suivent une formation formelle dans le supérieur. Données pondérées.

Source : Insee, enquêtes Emploi de 2013 à 2015.

Certificat d'aptitude professionnelle (CAP). En 2015, 34 \% des diplômes préparés par les entrants en apprentissage se situent au niveau bac +2 et plus, contre $13 \%$ dix ans auparavant (PESONEL, 2016). Dans le supérieur, l'apprentissage est particulièrement répandu dans les STS, les écoles d'ingénieurs et, dans une moindre mesure, les IUT (graphique 1).

Rares en premier cycle universitaire, les stages d'études ${ }^{10}$ sont plus fréquents au-delà et dans les formations à visée explicitement professionnalisante comme les licences et masters professionnels, les cursus d'ingénieurs, dans les écoles de commerce et en IUT (graphique 2). Les autres activités intégrées, en particulier celles de « salariés étudiants », concernent aussi des filières situées à un niveau d'études avancé (troisième cycle universitaire) et peuvent comporter l'exercice d'une activité rémunérée, pour certains obligatoire, dans des lieux et selon des conditions liées à la formation (graphique 2). C'est le cas par exemple des étudiants en troisième cycle de santé (médecine, pharmacie ou odontologie), qui travaillent au sein d'un centre hospitalier universitaire ou d'un cabinet agréé, ou des étudiants qui se destinent à l'enseignement ou à la recherche et occupent des postes d'Ater ou d'allocataires de recherche.

10. Hors stages d'entrée dans des métiers qualifiés du secteur public (médecins, infirmiers, enseignants, etc.) qui sont classés dans la catégorie « salariés étudiants ». 
Les activités occasionnelles non liées aux études, exercées l'été ou non, concernent plus souvent des étudiants jeunes et donc moins avancés dans leur cursus scolaire. À l'inverse, les activités régulières non liées aux études concernent des étudiants en moyenne plus âgés et inscrits plus souvent en lettres, art, langues étrangères, éducation et dans les formations préparant aux métiers de services ${ }^{11}$.

\section{Le temps consacré aux activités rémunérées : un temps intégré ou additionnel aux études}

Par rapport aux activités professionnelles non liées aux études, celles qui y sont liées sont exercées plus souvent en semaine, sur un plus grand nombre de jours et pour un temps de travail hebdomadaire plus long.

TABLEAU 2 - Durée et organisation du temps de travail selon le type d'activité exercée

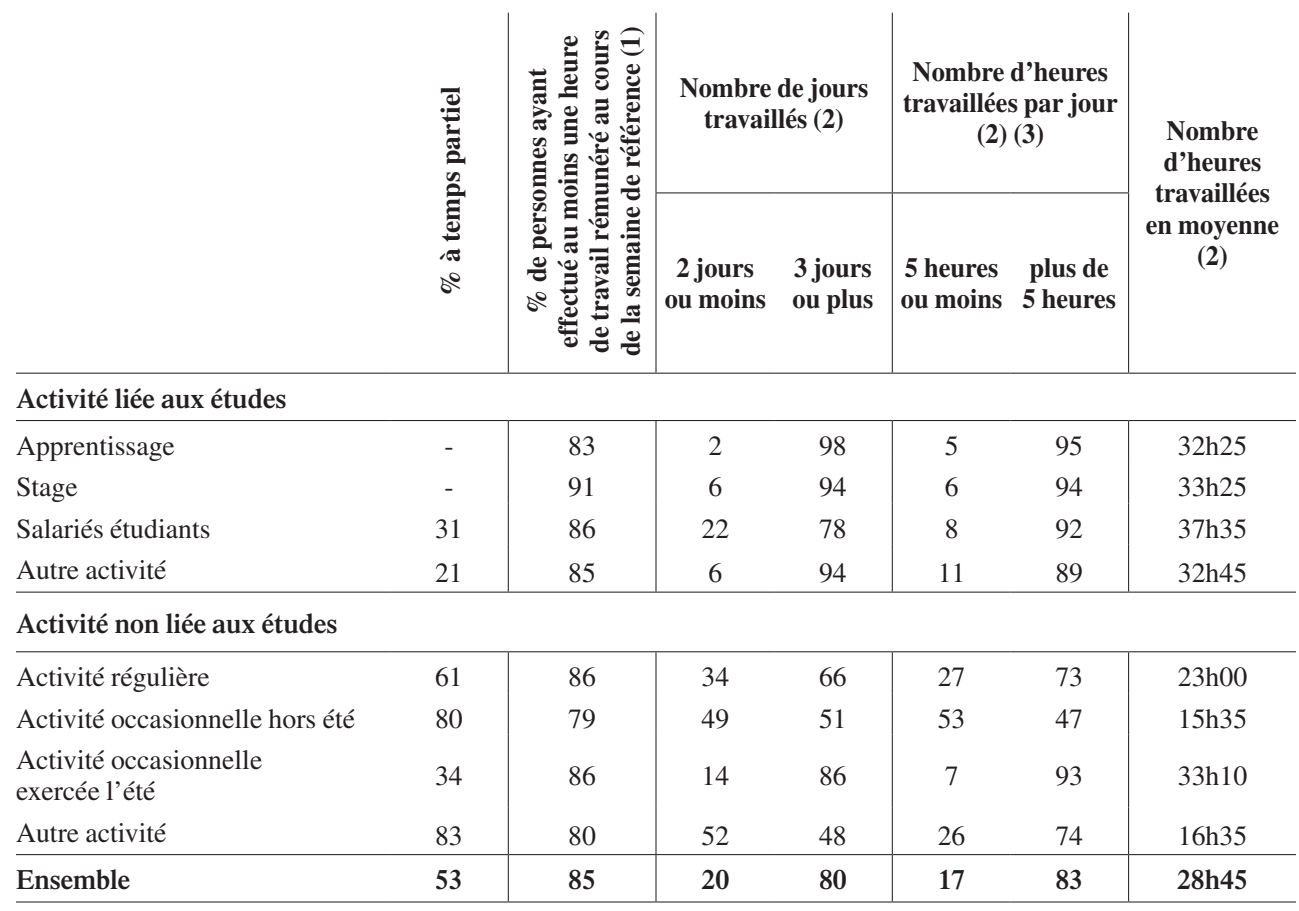

- : non concernés par le temps partiel.

(1) Les personnes absentes de leur emploi la semaine de référence le sont principalement pour cause de congé rémunéré ou de formation.

(2) Les durées sont calculées sur le champ des personnes ayant travaillé la semaine de référence.

(3) Heures hebdomadaires effectives rapportées au nombre de jours travaillés au cours d'une semaine de référence.

Lecture : en moyenne de 2013 à 2015, $61 \%$ des étudiants qui ont une activité régulière non liée aux études sont à temps partiel.

Champ : élèves ou étudiants âgés de 18 à 29 ans qui suivent une formation formelle dans le supérieur et occupent un emploi au moment de l'enquête. Données pondérées.

Source : Insee, enquêtes Emploi de 2013 à 2015.

11. La variable disponible dans l'enquête pour les années 2013 à 2015 ne permet pas de faire des regroupements différents de ceux proposés ici. 
Dans les périodes de travail, la durée de travail des apprentis est, en moyenne, la même que celle des autres employés de l'entreprise, avec un nombre de jours travaillés et un nombre d'heures par jour relativement élevés (tableau 2). Sur le lieu de travail, l'apprenti est soumis à des dispositions applicables à l'ensemble des salariés ainsi qu'à des dispositions particulières applicables aux jeunes travailleurs. Le temps consacré à la formation en centre de formation d'apprentis (généralement une semaine par mois) est considéré comme du temps de travail effectif dans le droit du travail.

Le stagiaire $^{12}$ travaille à temps complet, car il ne suit pas d'enseignements pendant sa période de stage. Sa présence dans l'entreprise ou l'organisme d'accueil suit les règles applicables aux salariés de l'entreprise pour ce qui concerne les durées maximales quotidiennes et hebdomadaires de présence, le travail de nuit, le repos quotidien, le repos hebdomadaire et les jours fériés. En conséquence, le nombre d'heures médian de travail est de 35 heures par semaine ( 5 jours par semaine et 7 heures par jour) (tableau 2). Les salariés étudiants, par rapport aux autres étudiants qui travaillent, effectuent un nombre élevé d'heures par jour (médiane de 8 heures sur 5 jours), travaillent souvent le samedi et le dimanche et, dans le cas des internes surtout, plus souvent tard le soir et la nuit (tableau 3).

TABLEAU 3 - Plages et jours de travail atypiques selon le type d'activité exercée

\begin{tabular}{|c|c|c|c|c|}
\hline \multirow[b]{2}{*}{ En pourcentages } & \multicolumn{4}{|c|}{ Au moins une fois au cours des $\mathbf{4}$ semaines précédant l'enquête... } \\
\hline & $\begin{array}{l}\text {...le soir (entre } \\
\text { 20h et minuit) }\end{array}$ & $\begin{array}{l}\text {...la nuit (entre } \\
\text { minuit et } 5 \mathrm{~h} \text { ) }\end{array}$ & ...le samedi & ...le dimanche \\
\hline \multicolumn{5}{|l|}{ Activité liée aux études } \\
\hline Apprentissage & 8 & 3 & 23 & 6 \\
\hline Stage & 16 & 5 & 14 & 7 \\
\hline Salariés étudiants & 52 & 31 & 57 & 41 \\
\hline Autre activité & 32 & 10 & 37 & 26 \\
\hline \multicolumn{5}{|l|}{ Activité non liée aux études } \\
\hline Activité régulière & 31 & 8 & 49 & 27 \\
\hline Activité occasionnelle hors été & 35 & 9 & 62 & 33 \\
\hline $\begin{array}{l}\text { Activité occasionnelle } \\
\text { exercée l'été }\end{array}$ & 25 & 11 & 55 & 33 \\
\hline Autre activité & 44 & 12 & 56 & 37 \\
\hline Ensemble & 48 & 51 & 40 & 22 \\
\hline
\end{tabular}

Lecture : en moyenne de 2013 à 2015, 49 \% des étudiants qui ont une activité régulière non liée aux études ont travaillé au moins un samedi sur une période de référence de 4 semaines au cours de l'année.

Champ : élèves ou étudiants âgés de 18 à 29 ans qui suivent une formation formelle dans le supérieur et occupent un emploi au moment de l'enquête. Données pondérées.

Source : Insee, enquêtes Emploi de 2013 à 2015.

12. Les stages sont identifiés à partir des déclarations des individus. Les stages d'entrée pour des métiers qualifiés du secteur public (médecins, infirmiers, enseignants, etc.) sont classés dans la catégorie « salariés étudiants ». 
Les activités occasionnelles occupées l'été (5\% des étudiants en emploi) sont plus souvent exercées à temps complet, plus de 3 jours par semaine et plus de 5 heures par jour. À l'inverse, les activités occasionnelles exercées en dehors des mois d'été (9\%) sont plus souvent exercées à temps partiel $(80 \%)$. En moyenne, les étudiants concernés travaillent 15 heures 35 minutes au cours de la semaine, concentrées le plus souvent sur moins de 3 jours (tableau 3 ).

Si la plupart des emplois réguliers sont occupés à temps partiel, ils représentent néanmoins le plus souvent une charge horaire lourde : $66 \%$ des étudiants concernés travaillent au moins 3 jours par semaine et $73 \%$ ont des journées de plus de 5 heures par jour travaillé, soit en moyenne 23 heures hebdomadaires. Ces emplois sont moins souvent exercés le soir ou le week-end, et moins souvent à mi-temps que les emplois occasionnels (tableau B, modèle 3, en annexe).

D'après la littérature, la fréquence des activités rémunérées, lorsqu'elles ne sont pas intégrées aux études, est un facteur déterminant dans la réussite aux examens, pour des raisons de temps disponible pouvant être consacré aux études (VouRC'H, 2009), d'où notre distinction entre activités non intégrées aux études exercées occasionnellement ou régulièrement.

\section{L'origine sociale des étudiants selon les filières d'études et les types d'activités rémunérées}

En France, l'accès à l'enseignement supérieur, de même que la composition des publics des différentes filières d'études, est fortement polarisé selon l'origine sociale (Albouy, WanecQ, 2003 ; Duru-Bellat, KiefFer, 2008). Les enfants dont le père est cadre ou a une profession intellectuelle supérieure sont surreprésentés dans l'enseignement supérieur (29\% des étudiants ont un père cadre ou de profession intellectuelle supérieure contre $17 \%$ des jeunes de 18 à 29 ans) et particulièrement dans les filières les plus élitistes (51\% des étudiants en CPGE, $46 \%$ dans la filière santé à l'université et en école de commerce, $42 \%$ en formation d'ingénieurs). À l'inverse, les enfants d'ouvriers sont sous-représentés dans l'enseignement supérieur (19\% ont un père ouvrier contre $32 \%$ des jeunes du même âge) et dans tous les types d'études. Ils sont un peu plus présents dans le segment professionnalisant (en BTS, en DUT, en apprentissage), qui propose des parcours d'études plus courts.

À l'exclusion de l'apprentissage, les activités non liées aux études, occasionnelles, exercées l'été ou non, ou régulières, ont en commun de concerner une population étudiante moins dotée socialement que celle exerçant une activité liée aux études (tableau 1). Les étudiants qui exercent une activité occasionnelle en dehors de l'été - plus souvent des filles - travaillent souvent le soir et le week-end (tableau 3). Les activités occasionnelles, exercées l'été ou non, concernent plus souvent de jeunes 
étudiants vivant au domicile de leurs parents (tableau B, modèles 2 et 3 , en annexe) ) $^{13}$ en «phase d'indépendance » ou d' « émancipation progressive », pour reprendre les termes de Louis GRUEL (1996). À l'inverse, les étudiants occupant des activités régulières non liées aux études sont en moyenne plus âgés, ont plus souvent un logement indépendant (tableau B, modèle 2) et vivent plus souvent en couple.

On l'a vu, filières d'études et types d'emplois occupés par les étudiants sont liés. Selon les caractéristiques des études, le cadre et le rythme de vie varient. Le rapport entre temps disponible et volume de travail à réaliser pour les études est donc plus ou moins équilibré. Les niveaux d'exigence, de structuration de l'emploi du temps et d'investissement distinguent les étudiants appartenant à chaque type d'études et confirment que « tous les étudiants ne sont pas étudiants au même degré » (GRIGNON, GruEL, 1999). Les étudiants en santé ont par exemple de plus fortes chances d'être « salariés étudiants » lorsqu'ils sont actifs ; en revanche, ils ont une probabilité très faible d'exercer des activités déconnectées des études (tableau B en annexe). Cela dessine la différenciation sociale importante qui existe entre les emplois, selon qu'ils sont liés aux études ou qu'ils entrent en concurrence avec elles. Les enfants de cadres (ou de professions intellectuelles supérieures) sont surreprésentés dans les activités intégrées aux études - en dehors de l'apprentissage, qui concerne une population plus mixte socialement - dans les emplois qualifiés (cadre, enseignant) et, dans une moindre mesure, dans les emplois très occasionnels (garde d'enfants), tandis que les enfants d'ouvriers sont surreprésentés parmi les employés de commerce (vendeurs, caissiers, etc.), les employés de services (serveurs, nettoyeurs, gardiens, aides à domicile, etc.) et les ouvriers. Cela suggère également qu'à travers la diversité des situations de travail, que l'activité soit exercée pendant l'été, de manière occasionnelle, ou bien de façon plus régulière au cours de l'année universitaire, les expériences de travail peuvent avoir des effets différenciés dans la vie de l'étudiant, entre ceux qui peuvent se consacrer pleinement à la poursuite de leurs études et les autres.

\section{Travailler et étudier, quels effets sur la poursuite des études selon les types d'emploi ?}

L'amélioration du taux de réussite est devenue progressivement une préoccupation centrale de la politique universitaire ${ }^{14}$. D'après l'OCDE (2016), environ un tiers des jeunes qui commencent une formation à l'université en France n'en sort pas au bout de trois ans avec un niveau de diplôme au moins équivalent à une licence. Ce taux ne

\footnotetext{
13. Les variables explicatives retenues sont le type, le niveau et la discipline des études, le sexe, le fait d'avoir un père cadre/profession intellectuelle supérieure ou non, et le fait de vivre ou non dans un logement indépendant de ses parents. 14. La loi Savary de 1984, la réforme Jospin/Lang ou encore la réforme Bayrou comptent parmi leurs objectifs la remontée des taux de réussite et la lutte contre les abandons. En 2008, le Plan pour la « réussite en Licence » de Valérie Pécresse ambitionnait de diviser par deux le taux d'échec en licence d'ici 2012.
} 
signifie cependant pas que tous ces étudiants sont en situation d'échec, puisqu'environ la moitié de ceux qui abandonnent leurs études universitaires se réoriente ensuite avec succès vers des formations plus courtes et techniques, type STS et IUT. Les parcours studieux des étudiants peuvent, en effet, être ponctués de réorientations (changements de formation ou d'établissement), d'interruptions ou d'abandons.

Afin d'étudier le lien entre activité et passage au niveau d'études supérieur (pour un panel d'étudiants interrogés au deuxième trimestre et réinterrogés au troisième et au quatrième trimestre de la même année), nous recourons à des modèles probit simples. Les variables communes utilisées dans les trois modèles reproduits dans le tableau 4 (pour tous les étudiants, puis seulement pour ceux qui sont en emploi) sont le type d'activité rémunérée, le type et le niveau d'études, la discipline des études, le sexe et la catégorie socioprofessionnelle du père ${ }^{15}$. Elles sont introduites dans la mesure où elles influencent les chances de réussite des étudiants. L'enquête Emploi ne permet pas de mesurer précisément le retard dans les études, mais on sait par exemple que les étudiants en première année de licence de droit, de lettres ou de sciences ont des taux de redoublement moins élevés que les étudiants de première année en santé, qui sont confrontés à des épreuves plus sélectives. On sait aussi que le fait de devenir étudiant et de le rester est inégalement probable selon les classes sociales (PINTO, 2014). Les étudiants dont le père est cadre ont plus de chances de succès que les autres, tandis que ceux dont le père est ouvrier présentent des probabilités de passage un peu plus faibles que ceux dont le père est employé, de profession intermédiaire ou indépendant et $a$ fortiori cadre - et ce, même lorsque les étudiants sont placés à égalité du point de vue de l'exercice d'une activité rémunérée (voir modèle 1, tableau 4). Ce résultat souligne l'importance des inégalités sociales d'apprentissage des étudiants dans le primaire et dans le secondaire, et leur impact sur les résultats des étudiants dans l'enseignement supérieur (Poullaouec, Hugrée, 2011).

D'après notre analyse (modèle 1, tableau 4), qui estime la probabilité de passer au niveau supérieur selon l'exercice ou non d'une activité rémunérée et, le cas échéant, le type d'activité, un étudiant qui exerce une activité liée à ses études a plus de chances de passer à un niveau d'études supérieur qu'un étudiant qui ne travaille pas, ou qu'un jeune qui exerce une activité occasionnelle ou régulière non liée à ses études, à autres caractéristiques introduites comparables. Les emplois en lien avec la formation, notamment parce qu'ils seraient plus valorisés par les étudiants et les employeurs, représenteraient moins souvent une entrave pour la réussite des études (BÉDUwÉ, GIRET, 2004).

Un deuxième modèle (modèle 2, tableau 4) est réalisé sur les seuls étudiants en emploi. Par rapport au modèle précédent, il introduit des informations sur la durée travaillée (plus ou moins d'un mi-temps) et sur les jours travaillés (la semaine, le week-end). D'après ce modèle, le fait d'avoir un père cadre ou ouvrier n'est pas significativement corrélé au fait de passer à un niveau supérieur d'études. Cela peut

15. Le retard scolaire aurait pu être une variable pertinente pour l'analyse. Cependant, il est difficilement mesurable car le niveau d'études atteint est trop imprécis dans l'enquête pour certains types d'études. 
TABLEAU 4 - Probabilités estimées de passer au niveau d'études supérieur : les effets du type d'activité (modèles probit simples)

$\begin{array}{ccc}\text { Modèle 2: } & \begin{array}{c}\text { Modèle 3: } \\ \text { les effets du }\end{array} \\ \begin{array}{c}\text { Modèle 1 : } \\ \text { les effets du } \\ \text { type d'activité }\end{array} & \begin{array}{c}\text { les effets du } \\ \text { type d'activité } \\ \text { (étudiants } \\ \text { en emploi) }\end{array} & \begin{array}{c}\text { et du nombre } \\ \text { d'heures } \\ \text { (étudiants } \\ \text { en emploi) }\end{array}\end{array}$

\begin{tabular}{|c|c|c|c|}
\hline Constante & $\begin{array}{l}0,47^{* * *} \\
(0,07)\end{array}$ & $\begin{array}{l}0,80^{* * * *} \\
(0,27) \\
\end{array}$ & $\begin{array}{l}0,94^{\text {**** }} \\
(0,30)\end{array}$ \\
\hline \multicolumn{4}{|c|}{ Type d'activité rémunérée exercée } \\
\hline Pas d'activité & Réf. & - & \\
\hline Activité liée aux études & $\begin{array}{c}0,20^{* * * *} \\
(0,07)\end{array}$ & $\begin{array}{l}0,32^{* *} \\
(0,15)\end{array}$ & \\
\hline Activité occasionnelle & $\begin{array}{l}-0,05 \\
(0,11)\end{array}$ & Réf. & \\
\hline Activité régulière & $\begin{array}{c}0,03 \\
(0,09)\end{array}$ & $\begin{array}{c}0,11 \\
(0,15)\end{array}$ & \\
\hline
\end{tabular}

Type d'activité rémunérée exercée et nombre d'heures de travail par semaine

Activité intégrée aux études

Réf.

Activité occasionnelle - moins de 10 heures par semaine

Activité occasionnelle - au moins 10 heures par semaine

$-0,19$

$(0,22)$

$-0,34^{* *}$

$(0,17)$

0,07

$(0,22)$

$-0,02$

$(0,23)$

$-0,40^{*}$

$(0,20)$

$-0,37^{* *}$

$(0,18)$

Activité régulière - plus de 28 heures par semaine

Temps de travail

\begin{tabular}{lcc}
\hline À mi-temps ou moins & \multicolumn{2}{c}{$-0,03$} \\
Plus d'un mi-temps & $(0,13)$ & \multicolumn{1}{c}{ Réf. } \\
\hline Travail la semaine du lundi au vendredi & $-0,37^{* *}$ & $-0,23$ \\
\hline Oui & $(0,15)$ & $(0,18)$ \\
Non & Réf. & Réf. \\
\hline Travail le week-end & $-0,11$ & $-0,12$ \\
\hline Oui & $(0,12)$ & $(0,12)$ \\
Non & Réf. & Réf. \\
\hline
\end{tabular}




\section{Type d'études et niveau}

STS

IUT

Université - $1^{\text {re }}$ année de Licence

Université - $2^{\mathrm{e}}$ année de Licence

Université - $3^{\mathrm{e}}$ année de Licence

Université - Master

École d'ingénieur ou de commerce

Discipline des études

Lettres, arts, langues étrangères, éducation

Sciences sociales, commerce et droit

Sciences, agriculture, industrie

Santé

Services

Programmes généraux, spécialité inconnue

\section{Sexe}

Homme

Femme

\section{CSP du père}

Père cadre/profession intellectuelle supérieure

Père ouvrier

Père retraité, au chômage, sans activité ou de profession inconnue

Père non cadre et non ouvrier

$\begin{array}{lll}0,45^{* * * *} & 0,64^{* * * *} & 0,66^{* * *} \\ (0,10) & (0,17) & (0,17) \\ 0,59^{* * * *} & 0,95^{* * *} & 0,97^{* * * *}\end{array}$

$(0,10) \quad(0,31) \quad(0,31)$

Réf. Réf. Réf.

$0,23^{* * *} \quad 0,51^{* * * *} \quad 0,51^{* *}$

$\begin{array}{lll}(0,07) & (0,21) & (0,21)\end{array}$

$0,10 \quad 0,33^{*} \quad 0,38 * *$

$(0,08) \quad(0,19) \quad(0,19)$

$\begin{array}{lll}0,00 & 0,29 & 0,29\end{array}$

$(0,08) \quad(0,19) \quad(0,20)$

$0,22^{* *} \quad 0,38^{*} \quad 0,40^{*}$

$\begin{array}{lll}(0,09) & (0,22) & (0,22)\end{array}$

$0,20^{* *} \quad 0,17 \quad 0,1$

$(0,08) \quad(0,22) \quad(0,22)$

Réf. Réf. Réf.

$0,17^{* *} \quad 0,12 \quad 0,13$

$(0,08) \quad(0,21) \quad(0,21)$

$-0,18^{* *} \quad-0,28 \quad-0,26$

$(0,08) \quad(0,24) \quad(0,24)$

$\begin{array}{lll}0,08 & 0,12 & 0,1\end{array}$

$(0,13) \quad(0,31) \quad(0,32)$

$\begin{array}{lll}-0,28 & -0,30 & -0,29\end{array}$

$\begin{array}{lll}(0,10) & (0,20) & (0,20)\end{array}$

$-0,06 \quad-0,07 \quad-0,06$

$(0,04) \quad(0,10) \quad(0,10)$

Réf. Réf. Réf.

Nombre d'observations

\begin{tabular}{ccc}
$0,12^{* * *}$ & 0,15 & 0,15 \\
$(0,05)$ & $(0,12)$ & $(0,12)$ \\
$-0,14^{* *}$ & 0,09 & 0,10 \\
$(0,06)$ & $(0,12)$ & $\left({ }^{*} 0,12\right)$ \\
0,10 & $0,42^{* *}$ & $0,42^{* *}$ \\
$(0,09)$ & $(0,22)$ & $(0,22)$ \\
Réf. & Réf. & Réf. \\
\hline 4369 & 907 & 907 \\
\hline
\end{tabular}

${ }^{* * * *}$ : significativement différent de 0 au seuil de $1 \%,{ }^{* *}:$ au seuil de $5 \%,{ }^{*}:$ au seuil de $10 \%$. Les coefficients sont issus de modélisations probit non pondérées. Les écarts-types, entre parenthèses, sont robustes à la présence d'hétéroscédasticité. La région du lieu d'habitation et l'âge (en classes), non présentés dans les tableaux, ont également été introduits dans les analyses à titre de contrôle.

Champ : élèves ou étudiants âgés de 18 à 29 ans qui suivent une formation formelle dans le supérieur, hors doctorat et CPGE, interrogés au $2^{\mathrm{e}}$ trimestre et réinterrogés aux $3^{\mathrm{e}}$ et $4^{\mathrm{e}}$ trimestres de la même année d'enquête.

Source : Insee, enquêtes Emploi de 2013 à 2015. 
tenir au fait qu' elle est fortement corrélée au type d'activité exercée : les étudiants dont le père est cadre (ou profession intellectuelle supérieure) travaillent significativement moins que ceux dont le père est d'une catégorie socio-professionnelle autre qu' ouvrier ou cadre (tableau B, modèle 1, en annexe). Et parmi les étudiants qui sont en emploi, le fait d'avoir un père ouvrier augmente la probabilité d'exercer une activité régulière plutôt qu'une activité liée aux études (tableau B, modèle 2, en annexe).

Pour les étudiants en emploi, la discipline des études ne paraît pas corrélée avec le fait de passer à un niveau d'études supérieur (modèles 2 et 3, tableau 4). Passer à un niveau d'études supérieur est en revanche corrélé avec le type et le niveau des études, avec l'activité professionnelle exercée et le fait de travailler ou non en semaine. Travailler durant la semaine réduit significativement la probabilité de passer au niveau supérieur, tandis que travailler le week-end ou non n'est pas significativement lié à ce passage. On peut penser à ce titre que ce qui peut être le plus préjudiciable à la réussite sont les horaires de travail, lorsqu'ils ne permettent pas aux étudiants d'assister aux enseignements. Cet effet disparaît si l'on utilise une variable croisant le type d'activité et le nombre d'heures travaillées par semaine (modèle 3). Par rapport à un étudiant qui exerce une activité intégrée aux études, ceux qui ont une activité occasionnelle au moins 10 heures par semaine ou une activité régulière équivalente à au moins un mi-temps (18 heures par semaine) auront des chances réduites de passer à un niveau d'études supérieur, à autres caractéristiques introduites comparables. Autrement dit, si l'activité rémunérée peut constituer un facteur de réussite (le cas des activités intégrées aux études) et améliorer les conditions de vie d'étudiants qui travaillent pour financer leurs études, prendre leur autonomie vis-à-vis de leurs parents ou encore acquérir une expérience professionnelle, elle peut représenter un risque pour la réussite scolaire si elle est déconnectée des études et exercée de façon intensive.

Besoin de financement, désir d'autonomie ou constitution d'une expérience professionnelle, les raisons avancées par les étudiants dans les enquêtes Conditions de vie de l'OVE pour justifier l'exercice d'une activité parallèle aux études sont nombreuses. Elles révèlent également la pluralité des conditions d'emploi et des profils (BELGHITH, 2015). L'emploi étudiant est en outre un salariat particulier aux formes plurielles. Les types d'emplois étudiants (en particulier leur lien avec les études ou non) sont fortement liés aux types d'études suivies, tandis que ces dernières varient selon les parcours scolaires et les caractéristiques sociales. Une différenciation sociale se dessine donc en creux entre les étudiants qui travaillent, selon que leur emploi est lié ou non aux études.

L'exercice d'une activité en cours d'études est plus fréquent qu'il y a dix ans (cette comparaison est possible grâce au travail d'É. Coudin et C. TAVAN (2008), réalisé à partir de la même source), principalement en raison du développement de l'apprentissage dans l'enseignement supérieur. L'emploi étudiant semble ainsi désormais plus 
lié aux études, bien que la part d'étudiants exerçant un emploi concurrent reste toujours importante.

Notre travail, qui tient compte de la multiplicité des conditions d'études et des profils étudiants, montre, grâce au suivi d'un panel d'étudiants, que tous les types d'activité ne sont pas pareillement corrélés avec la poursuite des études. Si certaines activités peuvent être bénéfiques à l'étudiant pour ses études, les plus déconnectées des études, exercées de façon régulière ou intense, peuvent avoir des répercussions non négligeables sur les conditions d'études et sur la réussite.

Les analyses mériteraient d'être reproduites par niveau et type d'études, et d'être enrichies d'informations sur les parcours des étudiants dans le secondaire, la qualité de l'orientation et les réorientations afin de confirmer ou de nuancer les conclusions obtenues. Ces résultats correspondent toutefois à ceux établis par ailleurs. M. DAGENAIS et ses co-auteurs (2000), Magali BefFy et ses co-auteurs (2009), C. BÉDuwé et J.-F. GIRET (2004) et Kady Marie-Danielle BoDy (2014) ont montré qu'un emploi sans lien avec les études avec des horaires lourds (seuil de 15 à 17 heures par semaine selon les études) a plus de chances de perturber la réussite. Notre analyse montre quant à elle que les étudiants exerçant une activité occasionnelle non liée aux études plus de 10 heures par semaine, et ceux exerçant une activité régulière non liée aux études au moins 18 heures par semaine, ont des chances réduites de passer à un niveau d'études supérieur. À partir du moment où elle se substitue au temps des études, l'activité rémunérée peut ainsi nuire au déroulement des études et fragiliser des publics étudiants moins favorisés. Elle pourrait aussi avoir pour effet de réduire l'assiduité des étudiants aux enseignements et l'utilisation des équipements qui leur sont réservés sur leur lieu d'études, comme des salles informatiques (BELGHITH, 2015). Or, une assiduité qui diminue au fil des mois peut entraîner à terme l'abandon ou le décrochage des études supérieures (BEAUPÈRE et al., 2007). 


\section{BibLIOGRAPHIE}

Albouy V., WANeCQ T. (2003), «Les inégalités sociales d'accès aux grandes écoles », Économie et statistique, $\mathrm{n}^{\circ} 361$, pp. 27-52.

BÉDUWÉ C., GIRET J.-F. (2004), « Le travail en cours d'études a-t-il une valeur professionnelle ? », Économie et statistique, $\mathrm{n}^{\circ}$ 378-379, pp. 55-83.

BefFy M., FougÈre D., MAurel A. (2009), « L'impact du travail salarié des étudiants sur la réussite et la poursuite des études universitaires », Économie et statistique, $\mathrm{n}^{\circ} 422$, pp. 31-50.

BeLGhith F. (2015), « L'activité rémunérée des étudiants. Une diversité de situations aux effets contrastés », OVE Infos, $\mathrm{n}^{\circ} 30$, avril.

Beaupère N., Chalumeau L., Gury N., Hugrée C. (2007), L'Abandon des études supérieures, Paris, La Documentation française.

Body K. M.-D. (2014), Étude micro-économétrique de l'impact du travail salarié étudiant sur la réussite à l'université, thèse de doctorat.

Castell L., Portela M., Rivalin R. (2016), «Les principales ressources des 18-24 ans. Premiers résultats de l'enquête nationale sur les ressources des jeunes », Insee Première, $\mathrm{n}^{\circ} 1603$, juin.

Coudin É., TAVAN C. (2008), « Deux étudiants du supérieur sur dix ont un emploi : le premier en lien avec ses études, l'autre pas », Insee Première, n 1204, juillet.

Dagenais, M., Montmarquette C., Parent D., Viennot-Briot N. (2000), « Travail pendant les études, performance scolaire et abandon ", Économie publique, vol 1, n ${ }^{\circ}$, pp. 145-192.

Dubet F. (1994), «Dimensions et figures de l'expérience étudiante dans l'Université de masse $»$, Revue française de sociologie, $\mathrm{n}^{\circ} 35$, pp. 511-532.

Duru-Bellat M., KIEFFER A. (2008), « Du baccalauréat à l'enseignement supérieur en France : déplacement et recomposition des inégalités », Population, vol. 63, n¹, pp. 123-157.

Duru-Bellat M., Verley E. (2009), «Les étudiants au fil du temps : contexte et repères institutionnels » in Gruel L., Galland O. et Houzel G. (dir.), Les Étudiants en France. Histoire et sociologie d'une nouvelle jeunesse, Presses universitaires de Rennes, pp. 195-219.

ERLICH V. (1998), Les Nouveaux Étudiants. Un groupe social en mutation, Paris, Armand Colin.

ECKERT H. (2009), «Étudier, travailler... les jeunes entre désir d'autonomie et contrainte sociale », Sociologie et Sociétés, vol 41, n 1, pp. 239-261.

Galland O. (1995), Le Monde des étudiants, Paris, Presses universitaires de France.

Galtier B., Minni C. (2015), « Emploi et chômage des 15-29 ans en 2014. Stabilisation des taux d'activité, d'emploi et de chômage », Dares Analyses, $\mathrm{n}^{\circ}$ 088, décembre.

GIRET J.-F. (2011), « L'activité rémunérée des étudiants », in Galland O., Verley E., Vourc'h R. (dir.), Les Mondes étudiants. Enquête Conditions de vie 2010, La Documentation française, pp. 207-216. 
Giret J.-F., VAn de Velde C., Verley É. (dir.) (2016), Les Vies étudiantes. Tendances et inégalités, La Documentation française.

Gonzalez-Demichel C., NAuze-Fichet E. (2003), « Les contours de la population active : aux frontières de l'emploi, du chômage et de l'inactivité », Économie et statistique, $\mathrm{n}^{\circ} 362$, pp. 85-103.

GRUEL L. (1996), « Les ressources des étudiants et leur évolution », Les Cahiers de l'OVE, nº 1.

Grignon C., Gruel L. (1999), La Vie étudiante, Paris, Presses universitaires de France.

GRUEL L. (2002), «Les conditions de réussite dans l'enseignement supérieur », OVE Infos, $\mathrm{n}^{\mathrm{o}} 2$, avril.

Gruel L., Thiphaine B. (2004), « Formes, conditions et effets de l'activité rémunérée des étudiants », Éducation et formations, n ${ }^{\circ}$ 67, mars, pp. 51-60.

ISSEHNANE S. (2011), « Le développement de l'apprentissage dans le supérieur : une évaluation empirique à partir de l'enquête génération 2001 », Travail et Emploi, n 125, pp. 27-39.

LAHIRE B. (1997), «Les Manières d'étudier », Les Cahiers de l'OVE, Paris, La Documentation française.

LAїв N. (2014), « La réussite des étudiants selon les difficultés financières et la perception d'une allocation d'études », Note d'information, $\mathrm{n}^{\circ} 14.05$, juillet.

Millet M. (2000), Les Étudiants de médecine et de sociologie à l'étude. Matrices disciplinaires, nature des savoirs, et pratiques intellectuelles : une analyse sociologique comparée des logiques sociales et cognitives du travail étudiant, Thèse de doctorat en sociologie et anthropologie, Université Lumière - Lyon 2.

Montmarquette C., Viennot-Briot N. et Dagenais M. (2007), "Dropout, School Performance and Working While in School: an Econometric Model With Heterogenous Preferences", The Review of Economics and Statistics, vol. 89, n 4, pp. 752-760.

OCDE (2016), Regards sur l'éducation 2016 : les indicateurs de l'OCDE, Éditions OCDE.

Ove (2016), Repères, décembre.

PARENT D. (2006), "Work While in High School in Canada: Its Labour Market and Educational Attainment Effects", Canadian Journal of Economics, vol. 39, n 4, pp. 1125-1150.

Pesonel E. (2016), «L'apprentissage en 2015. Nette reprise dans les entreprises de moins de 10 salariés et dans le secteur public », Dares Résultats, n $^{\circ} 075$, décembre.

Pinto V. (2014), À l'école du salariat. Les étudiants et leurs «petits boulots », Paris, Presses universitaires de France, coll. « Le lien social».

Poulet-Coulibando P. (2008), «Les études combinées avec les emplois par les jeunes : comparaison entre pays européens », Éducation et formations, n ${ }^{\circ} 78$, novembre, pp. 203-219.

Poullaouec T., Hugrée C. (2011), « Qui sont les étudiants d'origine populaire » in Galland O., Verley E., Vourc'h R. (dir.), Les Mondes étudiants. Enquête Conditions de vie 2010, La Documentation française, pp. 207-2016. 
REY O. (2009), « 1987-1997 : La France redécouvre ses universités » in Gruel L., Galland O., Houzel G. (dir.), Les Étudiants en France. Histoire et sociologie d'une nouvelle jeunesse, Presses universitaires de Rennes, pp. 125-170.

Rouaud P., Joseph O. (dir.) (2014), Quand l'école est finie. Premiers pas dans la vie active de la génération 2010, CÉREQ.

RuHm C. J. (1997), “Is High School Employment Consumption or Investment?”, Journal of Labor Economics, vol. 15, n ${ }^{\circ}$ 4, pp. 735-776.

ThiphaINE B. (2002), « Les étudiants et l'activité rémunérée », OVE Infos, n 1, février.

Vourc'H R. (2009), « Des vies diversement studieuses » in Gruel L., Galland O., Houzel G. (dir.), Les Étudiants en France. Histoire et sociologie d'une nouvelle jeunesse, Presses universitaires de Rennes, pp. 275-290.

ZiLLONIZ S. (2017), « Les activités rémunérées des étudiants : quelles formes et quelle organisation ? », Dares Analyses, n 046, juillet. 


\section{AnNeXes}

TABLEAU A - Différentes analyses sur le travail étudiant

\begin{tabular}{|c|c|c|c|}
\hline & Unité statistique & $\begin{array}{c}\text { Mode } \\
\text { d'administration } \\
\text { du questionnaire }\end{array}$ & Études \\
\hline $\begin{array}{l}\text { Enquête Conditions de vie } \\
\text { des étudiants OVE } \\
\text { (BELGHITH, 2015) }\end{array}$ & L'étudiant & auto-administré & $\begin{array}{l}\text { Formations universitaires } \\
\text { (IUT et ingénieurs), } \\
\text { classes supérieures de } \\
\text { lycée (CPGE et STS), } \\
\text { écoles d'ingénieurs non } \\
\text { universitaires, écoles de } \\
\text { commerce, gestion et } \\
\text { vente, écoles sous tutelle } \\
\text { du ministère de la Culture } \\
\text { et de la Communication } \\
\text { (en 2013) }\end{array}$ \\
\hline $\begin{array}{l}\text { Panel de bacheliers MESR } \\
\text { (LAÏB, 2014) }\end{array}$ & Génération de bacheliers & auto-administré & $\begin{array}{c}\text { Ensemble de } \\
\text { l'enseignement supérieur }\end{array}$ \\
\hline $\begin{array}{l}\text { Enquête Génération Céreq } \\
\text { (RouAud et al., 2014) }\end{array}$ & $\begin{array}{l}\text { Génération de sortants du } \\
\text { système éducatif }\end{array}$ & enquêteur & $\begin{array}{l}\text { Cohorte de sortants de } \\
\text { formation initiale (hors } \\
\text { sortants d'apprentissage) }\end{array}$ \\
\hline $\begin{array}{l}\text { Enquête Emploi de l'Insee } \\
\text { (Coudin et al., 2008) }\end{array}$ & $\begin{array}{l}\text { Personne de } 15 \text { ans ou plus au } \\
\text { sein d'un logement ordinaire } \\
\text { (hors communauté) dont c'est } \\
\text { la résidence principale }\end{array}$ & enquêteur & Études initiales \\
\hline $\begin{array}{l}\text { Enquête nationale sur } \\
\text { les ressources des jeunes } \\
\text { Drees et Insee } \\
\text { (CASTELL } \text { et al., 2016) }\end{array}$ & $\begin{array}{l}\text { Jeune âgé de } 18 \text { à } 24 \text { ans (au } \\
1^{\text {er }} \text { octobre } 2014 \text { ) résidant en } \\
\text { France (hors Mayotte) vivant } \\
\text { en ménage ordinaire ou en } \\
\text { communauté }\end{array}$ & enquêteur & Études initiales \\
\hline
\end{tabular}




\begin{tabular}{|c|c|c|c|c|c|}
\hline Travail étudiant & $\begin{array}{l}\text { Part d'étudiants } \\
\text { qui travaille }(\%)\end{array}$ & $\begin{array}{c}\text { Dont vacances } \\
\text { d'été }\end{array}$ & $\begin{array}{l}\text { Dont emploi } \\
\text { intégré aux } \\
\text { études }\end{array}$ & $\begin{array}{l}\text { Dont travail } \\
\text { occasionnel }\end{array}$ & $\begin{array}{l}\text { Dont travail } \\
\text { régulier }\end{array}$ \\
\hline $\begin{array}{l}\text { L'activité } \\
\text { rémunérée } \\
\text { pendant une } \\
\text { année }\end{array}$ & $\begin{array}{c}45 \% \text { pendant } \\
\text { l'année universitaire } \\
(2013)\end{array}$ & - & $\begin{array}{c}21 \% \text { en stage, } \\
\text { alternance ou qui } \\
\text { ont une activité } \\
\text { rémunérée dont } \\
\text { le contenu est } \\
\text { en lien avec les } \\
\text { études }\end{array}$ & $\begin{array}{l}16 \% \text { ont } \\
\text { une activité } \\
\text { rémunérée non } \\
\text { liée aux études } \\
\text { et exercée moins } \\
\text { d'un mi-temps } \\
(\text { «jobs ») }\end{array}$ & $\begin{array}{l}9 \% \text { ont } \\
\text { une activité } \\
\text { rémunérée } \\
\text { concurrente des } \\
\text { études exercée } \\
\text { au moins à } \\
\text { mi-temps }\end{array}$ \\
\hline $\begin{array}{l}\text { L'activité } \\
\text { rémunérée } \\
\text { pendant une } \\
\text { année }\end{array}$ & $\begin{array}{c}41 \% \text { pour les } \\
\text { Bac }+1 \text { à } 55 \% \text { pour } \\
\text { les Bac }+4 \text { (panel } \\
2008)\end{array}$ & $\begin{array}{c}11 \% \text { pour les } \\
\text { Bac }+1 \text { à } 17 \% \\
\text { pour les Bac }+4\end{array}$ & - & $\begin{array}{c}19 \% \text { pour les } \\
\text { Bac }+1 \text { à } 23 \% \\
\text { pour les } \mathrm{Bac}+4\end{array}$ & $\begin{array}{c}11 \% \text { pour les } \\
\text { Bac }+1 \text { à } 16 \% \\
\text { pour les Bac }+4\end{array}$ \\
\hline $\begin{array}{l}\text { Les emplois sur } \\
\text { l'ensemble de la } \\
\text { durée des études }\end{array}$ & $\begin{array}{l}68 \% \text { ont travaillé } \\
\text { pendant leurs études } \\
\text { (génération 2010) }\end{array}$ & - & $\begin{array}{l}7 \% \text { ont eu un } \\
\text { emploi régulier } \\
\text { en lien avec leurs } \\
\text { études ( } 42 \% \\
\text { des emplois } \\
\text { réguliers) }\end{array}$ & $\begin{array}{c}52 \% \text { ont eu } \\
\text { un emploi } \\
\text { occasionnel } \\
\text { pendant les } \\
\text { vacances ou } \\
\text { l'année scolaire }\end{array}$ & $\begin{array}{l}16 \% \text { ont eu un } \\
\text { emploi régulier } \\
\text { pendant l'année } \\
\text { scolaire (au } \\
\text { moins } 8 \mathrm{~h} \text { par } \\
\text { semaine tout au } \\
\text { long de l'année) }\end{array}$ \\
\hline $\begin{array}{l}\text { L'emploi au } \\
\text { cours d'une } \\
\text { semaine de } \\
\text { référence }\end{array}$ & $\begin{array}{l}19 \% \text { (enquêtes } \\
\text { 2004-2006) }\end{array}$ & $3 \%$ un job d'été & $\begin{array}{l}9 \% \text { en stage, } \\
\text { apprentissage } \\
\text { ou en emploi } \\
\text { pré-inséré }\end{array}$ & $\begin{array}{l}1 \% \text { ont un petit } \\
\text { boulot }\end{array}$ & $\begin{array}{l}7 \% \text { ont un } \\
\text { emploi d'attente, } \\
\text { concurrent ou } \\
\text { d'appoint }\end{array}$ \\
\hline $\begin{array}{l}\text { L'emploi au } \\
\text { cours d'une } \\
\text { semaine de } \\
\text { référence }\end{array}$ & $\begin{array}{l}23 \% \text { des jeunes } \\
\text { qui se déclarent en } \\
\text { cours d'études ont } \\
\text { travaillé la semaine } \\
\text { précédant l'enquête } \\
\text { (fin 2014) }\end{array}$ & - & $\begin{array}{l}9 \% \text { travaillent } \\
\text { en alternance, } \\
3 \% \text { en stage } \\
\text { rémunéré }\end{array}$ & $\begin{array}{l}11 \% \text { ont } \\
\text { d'autres activités } \\
\text { rémunérées }\end{array}$ & \\
\hline
\end{tabular}


TABLEAU B - Probabilités estimées d'exercer un type d'activité rémunérée pendant ses études (modèles probit multinomiaux)

\begin{tabular}{|c|c|c|c|c|c|c|c|c|c|}
\hline & $\begin{array}{r}\text { Mod } \\
\text { d }\end{array}$ & $\begin{array}{l}\text { e } 1 \text { : Prob } \\
\text { une activi } \\
\text { selon }\end{array}$ & $\begin{array}{l}\text { abilité d'e } \\
\text { é rémuné } \\
\text { on type }\end{array}$ & $\begin{array}{l}\text { xercer } \\
\text { rée }\end{array}$ & $\begin{array}{r}\text { Mod } \\
\text { d'exerc } \\
\text { d'ac } \\
\text { (étu }\end{array}$ & $\begin{array}{l}\text { le } 2 \text { : Prob } \\
\text { un type } \\
\text { ivité rému } \\
\text { iants en el }\end{array}$ & $\begin{array}{l}\text { bbilité } \\
\text { articulier } \\
\text { eérée } \\
\text { iploi) }\end{array}$ & \begin{tabular}{|} 
Modè \\
Probabili \\
une ac \\
occasio \\
(étudia \\
emplo \\
activités \\
aux ét
\end{tabular} & $\begin{array}{l}3 \text { : } \\
\text { d'avoir } \\
\text { vité } \\
\text { nelle } \\
\text { ts en } \\
\text { hors } \\
\text { tégrées } \\
\text { des) }\end{array}$ \\
\hline & 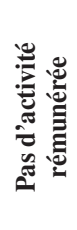 & 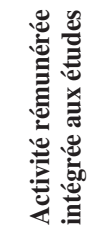 & 总 & 选 & 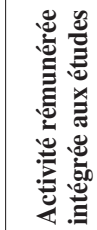 & 冚 & 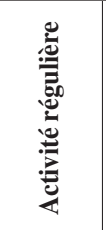 & 总 & 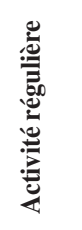 \\
\hline Constante & & $\begin{array}{c}-1,15^{* * * *} \\
(0,07)\end{array}$ & $\begin{array}{c}-1,98^{* * * *} \\
(0,10)\end{array}$ & $\begin{array}{c}-1,34^{* * * *} \\
(0,08)\end{array}$ & & $\begin{array}{c}-1,26^{* * * *} \\
(0,22)\end{array}$ & $\begin{array}{c}0,01 \\
(0,19)\end{array}$ & $\begin{array}{c}-1,38^{* * * *} \\
(0,23)\end{array}$ & \\
\hline
\end{tabular}

\begin{tabular}{|c|c|c|c|c|c|c|c|c|c|}
\hline \multicolumn{10}{|l|}{ Type d'études et niveau } \\
\hline STS & Réf. & $\begin{array}{l}0,60^{* * * *} \\
(0,07)\end{array}$ & $\begin{array}{c}0,13 \\
(0,10)\end{array}$ & $\begin{array}{c}0,06 \\
(0,09)\end{array}$ & Réf. & \begin{tabular}{|c|}
$-0,46^{* * * *}$ \\
$(0,16)$
\end{tabular} & $\begin{array}{c}-0,59^{* * * *} \\
(0,14)\end{array}$ & $\begin{array}{c}0,10 \\
(0,19)\end{array}$ & Réf. \\
\hline IUT & Réf. & $\begin{array}{c}-0,40^{* * * *} \\
(0,13)\end{array}$ & $\begin{array}{l}-0,02 \\
(0,14) \\
\end{array}$ & $\begin{array}{c}-0,46^{* * *} \\
(0,16)\end{array}$ & Réf. & $\begin{array}{c}0,32 \\
(0,27)\end{array}$ & $\begin{array}{l}-0,21 \\
(0,26) \\
\end{array}$ & $\begin{array}{l}0,56^{*} \\
(0,32)\end{array}$ & Réf. \\
\hline $\begin{array}{l}\text { Université - } 1^{\text {re }} \text { année de } \\
\text { Licence }\end{array}$ & Réf. & $\begin{array}{c}-1,38^{* * * *} \\
(0,20)\end{array}$ & $\begin{array}{l}-0,03 \\
(0,14) \\
\end{array}$ & $\begin{array}{c}-0,48^{* * *} \\
(0,15)\end{array}$ & Réf. & $\begin{array}{l}1,59^{* * * *} \\
(0,33)\end{array}$ & $\begin{array}{l}0,99^{* * *} \\
(0,33)\end{array}$ & $\begin{array}{l}0,56^{*} \\
(0,30)\end{array}$ & Réf. \\
\hline $\begin{array}{l}\text { Université - } 2^{\mathrm{e}} \text { année de } \\
\text { Licence }\end{array}$ & Réf. & $\begin{array}{c}-0,74^{* * * *} \\
(0,09)\end{array}$ & $\begin{array}{c}0,11 \\
(0,10)\end{array}$ & $\begin{array}{l}-0,08 \\
(0,09)\end{array}$ & Réf. & $\begin{array}{l}0,99^{* * *} \\
(0,19)\end{array}$ & $\begin{array}{l}0,90^{* * *} \\
(0,18)\end{array}$ & $\begin{array}{c}0,14 \\
(0,18)\end{array}$ & Réf. \\
\hline $\begin{array}{l}\text { Université - } 3^{\mathrm{e}} \text { année de } \\
\text { Licence }\end{array}$ & Réf. & $\begin{array}{l}-0,06 \\
(0,09)\end{array}$ & $\begin{array}{l}0,26^{* * *} \\
(0,10)\end{array}$ & $\begin{array}{l}0,20^{* *} \\
(0,09)\end{array}$ & Réf. & $\begin{array}{l}0,48^{* * * *} \\
(0,16)\end{array}$ & $\begin{array}{l}0,33^{* *} \\
(0,15)\end{array}$ & $\begin{array}{c}0,13 \\
(0,18)\end{array}$ & Réf. \\
\hline Université - Master & Réf. & Réf. & Réf. & Réf. & Réf. & Réf. & Réf. & Réf. & Réf. \\
\hline $\begin{array}{l}\text { École d'ingénieur ou de } \\
\text { commerce }\end{array}$ & Réf. & $\begin{array}{l}0,15^{*} \\
(0,09)\end{array}$ & $\begin{array}{c}-0,45^{* * *} \\
(0,14)\end{array}$ & \begin{tabular}{|c|}
$-1,04^{* * *}$ \\
$(0,15)$
\end{tabular} & Réf. & \begin{tabular}{|c|}
$-0,53 * *$ \\
$(0,21)$
\end{tabular} & $\begin{array}{c}-1,39^{* * *} \\
(0,22)\end{array}$ & $\begin{array}{l}1,00^{* * *} \\
(0,35)\end{array}$ & Réf. \\
\hline \multicolumn{10}{|l|}{ Discipline des études } \\
\hline $\begin{array}{l}\text { Université - Lettres, } \\
\text { arts, langues étrangères, } \\
\text { éducation }\end{array}$ & Réf. & $\begin{array}{l}-0,50^{* * * *} \\
(0,12)\end{array}$ & $\begin{array}{l}0,07 \\
(0,10)\end{array}$ & $\begin{array}{l}-0,01 \\
(0,10)\end{array}$ & Réf. & $\begin{array}{l}0,67^{* * *} \\
(0,20)\end{array}$ & $\begin{array}{l}0,51^{* * *} \\
(0,19)\end{array}$ & $\begin{array}{l}0,17 \\
(0,19)\end{array}$ & Réf. \\
\hline $\begin{array}{l}\text { Sciences sociales, } \\
\text { commerce et droit }\end{array}$ & Réf. & Réf. & Réf. & Réf. & Réf. & Réf. & Réf. & Réf. & Réf. \\
\hline $\begin{array}{l}\text { Sciences, agriculture, } \\
\text { industrie }\end{array}$ & Réf. & $\begin{array}{l}-0,12 \\
(0,11)\end{array}$ & $\begin{array}{c}-0,22^{*} \\
(0,12)\end{array}$ & $\begin{array}{c}-0,26^{* *} \\
(0,11)\end{array}$ & Réf. & $\begin{array}{l}-0,17 \\
(0,21)\end{array}$ & $\begin{array}{l}-0,19 \\
(0,20)\end{array}$ & $\begin{array}{c}0,02 \\
(0,24)\end{array}$ & Réf. \\
\hline Santé & Réf. & $\begin{array}{l}0,88^{* * *} \\
(0,12)\end{array}$ & $\begin{array}{l}-0,04 \\
(0,14) \\
\end{array}$ & $\begin{array}{c}-0,46^{* * *} \\
(0,16)\end{array}$ & Réf. & $\begin{array}{c}-1,05^{* * * *} \\
(0,24)\end{array}$ & $\begin{array}{c}-1,84^{* * * *} \\
(0,25)\end{array}$ & $\begin{array}{l}0,88^{* * * *} \\
(0,30)\end{array}$ & Réf. \\
\hline Services & Réf. & $\begin{array}{l}-0,09 \\
(0,17)\end{array}$ & $\begin{array}{l}-0,01 \\
(0,17)\end{array}$ & $\begin{array}{l}-0,09 \\
(0,16)\end{array}$ & Réf. & $\begin{array}{l}-0,12 \\
(0,35)\end{array}$ & $\begin{array}{l}-0,09 \\
(0,30)\end{array}$ & $\begin{array}{c}0,05 \\
(0,31)\end{array}$ & Réf. \\
\hline $\begin{array}{l}\text { Programmes généraux, } \\
\text { spécialité inconnue }\end{array}$ & Réf. & $\begin{array}{l}0,22^{* *} \\
(0,09)\end{array}$ & $\begin{array}{l}0,26^{* *} \\
(0,12)\end{array}$ & $\begin{array}{l}0,40^{* * * *} \\
(0,10)\end{array}$ & Réf. & $\begin{array}{c}0,23 \\
(0,19)\end{array}$ & $\begin{array}{l}0,30^{* *} \\
(0,15)\end{array}$ & $\begin{array}{l}-0,11 \\
(0,21)\end{array}$ & Réf. \\
\hline \multicolumn{10}{|l|}{ Sexe } \\
\hline Homme & Réf. & $\begin{array}{l}0,27^{* * *} \\
(0,05)\end{array}$ & $\begin{array}{c}-0,12^{* * *} \\
(0,06)\end{array}$ & $\begin{array}{l}0,10^{*} \\
(0,06)\end{array}$ & Réf. & $\mid \begin{array}{c}-0,32^{* * * *} \\
(0,10)\end{array}$ & $\begin{array}{l}-0,03 \\
(0,09)\end{array}$ & \begin{tabular}{|c}
$-0,35^{* * * *}$ \\
$(0,12)$
\end{tabular} & Réf. \\
\hline
\end{tabular}




\begin{tabular}{|c|c|c|c|c|c|c|c|c|c|}
\hline Femme & Réf. & Réf. & Réf. & Réf. & Réf. & Réf. & Réf. & Réf. & Réf. \\
\hline \multicolumn{10}{|l|}{ CSP du père } \\
\hline Père cadre & Réf. & \begin{tabular}{|c|}
$-0,17^{* * * *}$ \\
$(0,06)$
\end{tabular} & $\begin{array}{l}-0,14^{*} \\
(0,07)\end{array}$ & \begin{tabular}{|c|}
$-0,16^{* *}$ \\
$(0,07)$
\end{tabular} & Réf. & $\begin{array}{c}0,04 \\
(0,12)\end{array}$ & $\begin{array}{c}0,05 \\
(0,11)\end{array}$ & $\begin{array}{c}0,03 \\
(0,14)\end{array}$ & Réf. \\
\hline Père ouvrier & Réf. & $\begin{array}{c}0,04 \\
(0,06)\end{array}$ & $\begin{array}{c}0,01 \\
(0,08)\end{array}$ & $\begin{array}{l}0,23^{* * *} \\
(0,07)\end{array}$ & Réf. & $\begin{array}{c}0,04 \\
(0,13)\end{array}$ & $\begin{array}{l}0,30^{* *} \\
(0,12)\end{array}$ & $\begin{array}{l}-0,24 \\
(0,15)\end{array}$ & \\
\hline $\begin{array}{l}\text { Père retraité, au chômage, } \\
\text { sans activité ou de } \\
\text { profession inconnue }\end{array}$ & Réf. & $\begin{array}{c}-0,25^{* *} \\
(0,10)\end{array}$ & $\begin{array}{l}-0,17 \\
(0,12)\end{array}$ & $\begin{array}{l}0,03 \\
(0,10)\end{array}$ & Réf. & $\begin{array}{l}0,15 \\
(0,20) \\
\end{array}$ & $\begin{array}{l}0,43^{* *} \\
(0,17)\end{array}$ & $\begin{array}{l}-0,23 \\
(0,22)\end{array}$ & \\
\hline $\begin{array}{l}\text { Père non cadre et non } \\
\text { ouvrier }\end{array}$ & Réf. & Réf. & Réf. & Réf. & Réf. & Réf. & Réf. & Réf. & Réf. \\
\hline \multicolumn{10}{|l|}{ Logement } \\
\hline Vit chez ses parents & Réf. & \begin{tabular}{|c|}
$-0,75^{* * * *}$ \\
$(0,49)$ \\
\end{tabular} & \begin{tabular}{|c|}
$-0,32^{* * *}$ \\
$(0,06)$ \\
\end{tabular} & \begin{tabular}{|c|}
$-0,97^{* * * *}$ \\
$(0,06)$ \\
\end{tabular} & Réf. & $\begin{array}{l}0,41^{* * * *} \\
(0,10)\end{array}$ & \begin{tabular}{|c|}
$-0,41^{* * *}$ \\
$(0,09)$ \\
\end{tabular} & $\begin{array}{l}0,88^{* * * *} \\
(0,12) \\
\end{array}$ & Réf. \\
\hline $\begin{array}{l}\text { Ne vit pas chez ses } \\
\text { parents }\end{array}$ & Réf. & Réf. & Réf. & Réf. & Réf. & Réf. & Réf. & Réf. & Réf. \\
\hline \multicolumn{10}{|l|}{ Trimestre d'interrogation } \\
\hline $1^{\mathrm{er}}$ trimestre & Réf. & Réf. & Réf. & Réf. & Réf. & Réf. & Réf. & Réf. & Réf. \\
\hline $2^{\mathrm{e}}$ trimestre & Réf. & $\begin{array}{l}0,18^{* * * *} \\
(0,07)\end{array}$ & $\begin{array}{c}0,10 \\
(0,09)\end{array}$ & $\begin{array}{l}0,17^{* *} \\
(0,08)\end{array}$ & Réf. & $\begin{array}{c}0,1 \\
(0,14)\end{array}$ & $\begin{array}{c}0,12 \\
(0,12)\end{array}$ & $\begin{array}{l}-0,04 \\
(0,17)\end{array}$ & Réf. \\
\hline $3^{\mathrm{e}}$ trimestre & Réf. & $\begin{array}{l}-0,07 \\
(0,07)\end{array}$ & $\begin{array}{l}0,48^{* * *} \\
(0,08)\end{array}$ & $\begin{array}{c}0,04 \\
(0,08)\end{array}$ & Réf. & $\begin{array}{l}0,96^{* * *} \\
(0,14)\end{array}$ & $\begin{array}{l}0,26^{* *} \\
(0,13)\end{array}$ & $\begin{array}{l}0,76^{* * *} \\
(0,16)\end{array}$ & Réf. \\
\hline $4^{\mathrm{e}}$ trimestre & Réf. & $\begin{array}{c}-0,12^{*} \\
(0,07) \\
\end{array}$ & $\begin{array}{l}-0,14 \\
(0,09)\end{array}$ & $\begin{array}{l}-0,05 \\
(0,07)\end{array}$ & Réf. & $\begin{array}{c}0,00 \\
(0,15)\end{array}$ & $\begin{array}{c}0,13 \\
(0,12)\end{array}$ & $\begin{array}{l}-0,10 \\
(0,17)\end{array}$ & Réf. \\
\hline \multicolumn{10}{|l|}{ Temps de travail (1) } \\
\hline À mi-temps ou moins & & & & & Réf. & $\begin{array}{l}1,74^{* * * *} \\
(0,13)\end{array}$ & $\begin{array}{l}1,36^{* * *} \\
(0,13)\end{array}$ & $\begin{array}{l}0,44^{* * * *} \\
(0,13)\end{array}$ & Réf. \\
\hline Plus d'un mi-temps & & & & & Réf. & Réf. & Réf. & Réf. & Réf. \\
\hline \multicolumn{10}{|l|}{$\begin{array}{l}\text { Travail la semaine du } \\
\text { lundi au vendredi (1) }\end{array}$} \\
\hline Oui & & & & & Réf. & \begin{tabular}{|c|}
$-0,78^{* * * *}$ \\
$(0,16)$ \\
\end{tabular} & \begin{tabular}{|c|}
$-0,92^{* * * *}$ \\
$(0,14)$ \\
\end{tabular} & $\begin{array}{c}0,11 \\
(0,14)\end{array}$ & Réf. \\
\hline Non & & & & & Réf. & Réf. & Réf. & Réf. & Réf. \\
\hline \multicolumn{10}{|l|}{ Travail le week-end (1) } \\
\hline Oui & & & & & Réf. & $\begin{array}{l}0,95^{* * *} \\
(0,11)\end{array}$ & $\begin{array}{l}0,62^{* * * *} \\
(0,10)\end{array}$ & $\begin{array}{l}0,32^{* * * *} \\
(0,12)\end{array}$ & Réf. \\
\hline Non & & & & & Réf. & Réf. & Réf. & Réf. & Réf. \\
\hline Nombre d'observations & \multicolumn{4}{|c|}{10290} & \multicolumn{3}{|c|}{2277} & \multicolumn{2}{|c|}{1114} \\
\hline \multicolumn{10}{|c|}{$\begin{array}{l}\text { **** } \text { significativement différent de } 0 \text { au seuil de } 1 \%,{ }^{* *} \text { au seuil de } 5 \%, * \text { au seuil de } 10 \% \text {. Les coefficients sont issus d'une modélisation } \\
\text { probit multinomiale (effectif non pondéré, observations lors du premier trimestre d'interrogation). Les écarts-types, entre parenthèses, } \\
\text { sont robustes à la présence d'hétéroscédasticité. } \\
\text { (1) au cours de la semaine de référence. } \\
\text { Champ modèle } 1 \text { : élèves ou étudiants âgés de } 18 \text { à } 29 \text { ans qui suivent une formation formelle dans le supérieur, hors doctorat et hors } \\
\text { classes préparatoires aux grandes écoles. } \\
\text { Champ modèle } 2 \text { : élèves ou étudiants âgés de } 18 \text { à } 29 \text { ans qui suivent une formation formelle dans le supérieur et occupent un emploi } \\
\text { au moment de l'enquête, hors doctorat et hors classes préparatoires aux grandes écoles. } \\
\text { Champ modèle } 3 \text { : élèves ou étudiants âgés de } 18 \text { à } 29 \text { ans qui suivent une formation formelle dans le supérieur et occupent un emploi } \\
\text { au moment de l'enquête, hors doctorat et hors classes préparatoires aux grandes écoles, et hors activités intégrées aux études. } \\
\text { Source : Insee, enquêtes Emploi de } 2013 \text { à } 2015 \text {. }\end{array}$} \\
\hline
\end{tabular}

